\title{
Simulation of cell-substrate traction force dynamics in response to soluble factors
}

Tao Liu

\author{
Centre for Structural Engineering and Informatics \\ Department of Civil Engineering \\ Faculty of Engineering, University of Nottingham, \\ University Park, Nottingham, NG7 2RD, U.K. \\ Email: Tao.Liu@nottingham.ac.uk, Tel: +44 (0) 1157484059 \\ February 2017
}

\begin{abstract}
Finite element (FE) simulations of contractile responses of vascular muscular thin films (vMTFs) and endothelial cells resting on an array of micro-posts under stimulation of soluble factors were conducted in comparison with experimental measurements reported in literature. Two types of constitutive models were employed in the simulations, i.e. smooth muscle cell type and non-smooth muscle cell type. The time histories of the effects of soluble factors were obtained via calibration against experimental measurements of contractile responses of tissues or cells. The numerical results for vMTFs with micropatterned tissues suggest that the radius of curvature of vMTFs under stimulation of soluble factors is sensitive to width of the micropatterned tissue, i.e. the radius of curvature increases as the tissue width decreases. However, as the tissue response is essentially isometric, the time history of the maximum principal stress of the micropatterned tissues is not sensitive to tissue width. Good agreement has been achieved for predictions of the vasoconstrictor endothelin-1 (ET-1) induced contraction stress between the FE numerical simulation and the experiment based approach of Alford, et al. (2011) for the vMTFs with 40, 60, 80 and $100 \mu \mathrm{m}$ width patterns. This may suggest the contraction stress is weakly sensitive to the tissue width for these patterns. However, for $20 \mu \mathrm{m}$ width tissue patterning, the numerical simulation result for contraction stress is less than the average value of experimental measurements, which may suggest the thinner and more elongated spindlelike cells within the $20 \mu \mathrm{m}$ width tissue patterning have higher contractile output. The constitutive model for nonsmooth muscle cells was used to simulate the contractile response of the endothelial cells. The substrate was treated as an effective continuum. For agonists such as Lysophosphatidic acid (LPA) and vascular endothelial growth factor (VEGF), the deformation of the cell diminishes from edge to centre and the central part of the cell is essentially under isometric state. Numerical studies demonstrated the scenarios that cell polarity can be triggered via manipulation of the effective stiffness and Possion's ratio of the substrate.
\end{abstract}

Keywords: Cytoskeletal contractility; stress fibres; cell shape; finite element; bio-chemo-mechanical modelling 


\section{Introduction}

The phenomena of cell-substrate interaction have been widely used to examine the effect of cellular microenvironment in regulating cytoskeletal contractility as well as measure cellular contractile responses. The interaction forces are generated from cytoskeletal contractility and transmitted to the external extracellular matrix (ECM) through cross-bridging interactions between the actin filaments and myosin II motors, which form stress fibres, as well as the interactions between stress fibres and focal adhesions. During these interaction events, both external insoluble factors and internal soluble factors play certain roles. Significant experimental progress has been made to investigate the effects of both insoluble and soluble factors, such as topology and stiffness of substrates, adhesion-mediated signals as well as soluble vasoactive substances. For example, by seeding the cells on an array of poly (dimethylsiloxane) (PDMS) micro-posts, Tan et al. (2003) has measured the distribution of traction force exerted by single cells via deflections of the micro-posts. Yang et al. (2011) has employed the same technique to measure the traction force of single endothelial cells exposed to contractility soluble factors such as vascular endothelial growth factor and lysophosphatidic acid. To examine the effect of cell shape, Alford et al. (2011) has used the vascular muscular thin films (vMTFs) method to measure the contractile response of micropatterned tissues with widths ranging from $20 \mu \mathrm{m}$ to $100 \mu \mathrm{m}$. They have found that the more elongated spindle cell shape correlates to higher contractile output.

Numerical predictive models have been developed for facilitating the development of novel experimental approaches as well as interpretation of experimental results. For example, Deshpande et al. (2006) has developed a finite element based whole cell model that includes the events of stress fibres formulation and the cross-bridge cycling between the actin and the myosin filament. Based on the model, McGarry et al. (2009) has developed the numerical simulation on the experiments reported by Tan et al. (2003) with good agreement, which includes the contractile response of smooth muscle cells, mesenchymal stem cells and fibroblasts resting on an array of PDMS microposts. ObbinkHuizer et al. (2014) has simulated local stress fibre orientation in uniaxially and biaxially constrained microtissues using a finite element model. In comparison with experimental measurement, they observed a strong local stress fibre alignment near the boundary of the tissues. Liu (2014) has recently extended the Hai-Murphy model (Hai and Murphy, 1988a) to include the strain rate effect on the kinetics of cross-bridge for isotonic contraction of smooth muscles. Combined with the 'characteristic equation' of Hill (1938), the new model has been used to simulate 
shortening-induced deactivation for smooth muscles with good agreement with experimental measurement. Loosli et al. (2010) has developed a predictive model to simulate cytoskeletal remodeling induced by cell spreading on micro-patterned islands. The results obtained from the model show close similarity to the experimental observation of epithelial cells spreading on micro-patterned substrates.

The numerical study reported in this paper focus on the effect of soluble factors on cell-substrate traction force dynamics. Soluble factors such as agonists can facilitate the release of calcium ion $\left(\mathrm{Ca}^{2+}\right)$ from intracellular stores or promote phosphorylation of myosin light chain II or actin polymerization. Although numerical simulations have been conducted towards understanding the regulation effect of external insoluble factors such as substrate shape and boundary conditions (McGarry et al., 2009; Obbink-Huizer et al., 2014), there are limited numerical studies to investigate the integrative effect of insoluble and soluble factors in regulating cytoskeletal contractility. This paper aims to provide insights into the integrative effect via numerical simulations on experimental studies reported by Alford et al. (2011) for vMTFs and Yang et al. (2011) for endothelial cells resting on an array of microposts, respectively.

\section{The constitutive models for cytoskeletal contractility}

In this paper, two types of cells are considered separately owing to distinct bio-chemo-mechanical features involved in cytoskeletal contractility, i.e. smooth muscle cells (SMCs) and non-smooth muscle cells (non-SMCs). SMCs can maintain steady-state contraction at relatively low level energy consumption required by myosin light chain phosphorylation and cross-bridge cycling. This phenomenon has been termed as 'latch state' in literature. Hai and Murphy (1988a) has developed a bio-chemical model accounting for the four myosin states involved in isometric contraction of a smooth muscle. The model is based on the assumption that latch-bridge and cross-bridge coexist in the system: the latch-bridge is generated by the dephosphorylation of attached phosphorylated myosin and the crossbridge by the attachment of phosphorylated myosin. Using the model, the calcium ion stimulation can be related to the kinetics of the fraction of myosin in the different states. Motivated by the experimental study of Barany (1967) that demonstrated that ATPase activity of myosin is proportional to speed of muscle shortening, Liu (2014) has extended the Hai-Murphy model for isotonic contraction of a single stress fibre by incorporating the effect of strain rate, which reads 


$$
\begin{aligned}
& \frac{d[M]}{d t}=-k_{1}[M]+k_{2}[M P]+\left(k_{7}-k_{9} \frac{k_{10}}{\xi} \frac{\dot{\varepsilon}}{\dot{\varepsilon}_{o}}\right)[A M] \\
& \frac{d[M P]}{d t}=\left(-k_{8} \frac{k_{10}}{\xi} \frac{\dot{\varepsilon}}{\dot{\varepsilon}_{o}}+k_{4}\right)[A M P]+k_{1}[M]-\left(k_{2}+k_{3}\right)[M P] \\
& \frac{d[A M P]}{d t}=k_{3}[M P]+k_{6}[A M]-\left(k_{4}+k_{5}-k_{8} \frac{k_{10}}{\xi} \frac{\dot{\varepsilon}}{\dot{\varepsilon}_{o}}\right)[A M P] \\
& \frac{d[A M]}{d t}=k_{5}[A M P]-\left(k_{6}+k_{7}-k_{9} \frac{k_{10}}{\xi} \frac{\dot{\varepsilon}}{\dot{\varepsilon}_{o}}\right)[A M]
\end{aligned}
$$

where $t$ denotes time; $[\mathrm{M}],[\mathrm{MP}],[\mathrm{AMP}]$ and $[\mathrm{AM}]$ denote fractions of the four myosin states, i.e. free unphosphorylated, phosphorylated, attached phosphorylated and attached unphosphorylated myosin, respectively, with $[\mathrm{M}]+[\mathrm{MP}]+[\mathrm{AMP}]+[\mathrm{AM}]=1 ; \xi$ the fraction of phosphorylated myosin, $\xi=[M P]+[A M P] ; \mathrm{k}_{i}(i=1, \ldots, 10)$ the rate constants with assumption that $k_{1}=k_{6}$ and $k_{2}=k_{5}$ (Hai \& Murphy, 1988a) $; \dot{\varepsilon}\left(\dot{\varepsilon}_{\max } \leq \dot{\varepsilon} \leq 0\right)$ and $\dot{\varepsilon}_{o}$ $\left(\dot{\varepsilon}_{o}>0\right)$ the isotonic shortening strain rate and a reference strain rate, respectively, where $\dot{\varepsilon}_{\text {max }}$ denotes the maximal shortening strain rate, i.e. the strain rate under unloaded contraction. Amongst all rate constants employed in equations (1) to (4), $k_{1}$ and $k_{6}$ are the only $\mathrm{Ca}^{2+}$-regulated rate constants and obtained through calibration of the model behaviour against experimental measurements; the rate constants $k_{8}$ and $k_{9}$ reflect the strain rate effect of isotonic contraction state with $k_{8}=k_{9}=0$ representing negligible influence of strain rate on the kinetic equations. Liu (2014) has applied the above mentioned model to simulate shortening-induced deactivation of smooth muscles. Calibrated against experimental measurements, the values of $k_{8}$ and $k_{9}$ varied from 1.7 to 16 for selected smooth muscles : a molluscan smooth muscle and an Aplysia I2 smooth muscle.

For non-smooth muscle type cells such as endothelial cells considered in the paper, two biochemical events are included in the biochemical model, i.e. (i) $\mathrm{Ca}^{2+}$ stimulation to promote phosphorylation of myosin light chain II and (ii) the phosphorylated myosin entering the crossbridge cycle to generate contraction forces, as shown in Fig. 1. The myosin molecules have three states (Murphy, 1988), i.e. free unphosphorylated, phosphorylated and attached phosphorylated states. The kinetics of the fraction of myosin can be given in the following kinetic equations

$$
\frac{d[M]}{d t}=-k_{1}[M]+k_{2}[M P]
$$




$$
\frac{d[M P]}{d t}=\left(-k_{8} \frac{k_{10}}{\xi} \frac{\dot{\varepsilon}}{\dot{\varepsilon}_{o}}+k_{4}\right)[A M P]+k_{1}[M]-\left(k_{2}+k_{3}\right)[M P]
$$

and

$$
\frac{d[A M P]}{d t}=k_{3}[M P]-\left(k_{4}-k_{8} \frac{k_{10}}{\xi} \frac{\dot{\varepsilon}}{\dot{\varepsilon}_{o}}\right)[A M P]
$$

where strain rate effect is included for isotonic contraction. Amongst rate constants employed in equations (5) to (7), $k_{1}$ is again the $\mathrm{Ca}^{2+}$-regulated rate constant and $k_{3}$ is the rate constant for attachment of myosin to actin filaments. The mechanical model of a single stress fibre is given as the stress strain rate relations for isotonic and eccentric contraction (Liu, 2014)

$$
\begin{array}{ll}
\frac{\sigma}{\sigma_{o}}=\frac{1+\left(k_{10} / \xi\right)\left(\dot{\varepsilon} / \dot{\varepsilon}_{o}\right)}{1-(1 / \xi)\left(\dot{\varepsilon} / \dot{\varepsilon}_{o}\right)}, & \text { if }-\frac{\xi}{k_{10}} \leq \frac{\dot{\varepsilon}}{\dot{\varepsilon}_{o}} \leq 0 \text { (isotonic contraction) } \\
\frac{\sigma}{\sigma_{o}}=1+\frac{\left(1+k_{10}\right)}{\xi} \frac{\dot{\varepsilon}}{\dot{\varepsilon}_{o}}, & \text { if } \frac{\dot{\varepsilon}}{\dot{\varepsilon}_{o}}>0 \quad \text { (eccentric contraction) }
\end{array}
$$

where $\sigma_{o}$ denotes the isometric stress of the stress fibre, which can be related to the fraction of attached phosphorylated and attached unphosphorylated myosin $\eta=[A M]+[A M P]$ for SMCs or attached phosphorylated myosin $\eta=[A M P]$ for non-SMCs, via

$$
\sigma_{o}=\frac{\sigma_{\max }}{[\eta]_{\max }} \eta
$$

where $\sigma_{\max }$ is the maximal tension under the maximal fraction of the attached myosin molecules, i.e. $[\eta]_{\max }=\max ([\mathrm{AMP}]+[\mathrm{AM}])$ for SMCs or $[\eta]_{\max }=\max ([\mathrm{AMP}])$ for non-SMCs, which is permitted by biochemistry (Hai and Murphy, 1988b).

The constitutive model for a single stress fibre described in Equations (1) through (9) can be extended to model two dimensional cytoskeletal networks through homogenisation over a representative volume element (RVE), as schematically shown in Fig. 2.The RVE is with cylindrical shape of radius $R$, height $\delta$ and local coordinates $\mathrm{y}_{1}-\mathrm{y}_{2}$. By assuming that $m$ stress fibres can form in any direction in two dimensional space with equal possibility in the 
RVE (Liu, 2014), the macroscopic Cauchy stress, $\overline{\boldsymbol{\sigma}}$, and rate of deformation, $\overline{\mathbf{D}}$, can be related to the Cauchy stress, $\sigma^{(k)}$, and the true strain rate, $\dot{\varepsilon}^{(k)}$, for the $k$ th stress fibre within the RVE, via

$$
\begin{aligned}
& \bar{\sigma}_{i j}=\sum_{k=1}^{m} c^{(k)} \sigma^{(k)} n_{i}^{(k)} n_{j}^{(k)}+\sigma_{p i j} \\
& \bar{\sigma}_{i j}=\frac{1}{\pi} \int_{-\pi / 2}^{\pi / 2} \sigma n_{i} n_{j} d \theta+\sigma_{p i j} \quad \text { if } m \rightarrow \infty \\
& \left.\dot{\varepsilon}^{(k)}=\bar{D}_{i j} n_{i}^{(k)} n_{j}^{(k)} \quad \text { (summation over } i \text { and } j ; i, j=1,2\right)
\end{aligned}
$$

where $c^{(k)}$ denotes the volume fraction of the $k$ th stress fibre in the current configuration with the orientation of the stress fibre aligned with unit vector $\mathbf{n}^{(k)}=n_{j}^{(k)} \mathbf{e}_{j} ; \theta$ is the angle between a stress fibre and local $\mathrm{y}_{1}$-axis of the RVE, see Fig. 2; $m$ is the total number of stress fibres in the RVE; and $\boldsymbol{\sigma}_{p}$ the passive stress, accounting for the contribution of intermediate filaments, nuclei and cell membrane to the material response. As the focus of the paper is on active responses of cytoskeletons, the passive component is simplified as a neo-Hookean like solid, see Supplementary Data for description of the model. The constitutive models for SMCs and non-SMCs have been implemented into the finite element package ABAQUS ${ }^{\circledR}$ Standard via user defined subroutine (UMAT). In the following, these constitutive models will be used to simulate cell-substrate traction force dynamics in response to soluble factors.

\section{Simulation of vascular muscular thin films (vMTFs)}

Vascular muscular thin films (vMTFs) have been used to examine the effect of cell shape on contractility of vascular SMCs (Alford et al., 2011). In this technique, extracellular matrix (ECM) proteins such as fibronectin or laminin were microcontact printed onto a polydimethylsiloxane (PDMS) layer as long bands. When seeded with vascular SMCs, cells were constrained to the ECM patterned portion of the PDMS layer, forming patterned tissues with widths ranging from $20 \mu \mathrm{m}$ to $100 \mu \mathrm{m}$. The contractile output of vMTFs can be measured via their radii of curvature. As the cells within micropatterned tissues could be forced into a more exaggerated spindle-like configuration in the thinner tissues, Alford, et al. (2011) has found that the elongated spindle cell shape strongly influences contractility. In this paper, the experimental study is simulated using the SMCs based constitutive model.

\section{The finite element model}


The finite element (FE) model of the vMTFs with micropatterned tissues is schematically shown in Fig. 3(a) at a deformed configuration. The micropatterned tissues with width $b$ and spacing $d$ are arranged on the top surface of the PDMS substrate. The length and width of the vMTFs are $L=2 \mathrm{~mm}$ and $w=1 \mathrm{~mm}$, respectively, which are sufficient wide that the stress calculation is independent of the size of the film. Throughout Section 3, coordinates X$\mathrm{Y}$ are defined with $\mathrm{X}$-axis representing the longitudinal direction of the vMTFs and Y-axis the width direction. As the geometry and the deformation of vMTFs are symmetric along the longitudinal direction, only half of the vMTFs is shown in Fig. 3 with the location of $\mathrm{X}=0$ corresponding to the free end and $\mathrm{X}=\mathrm{L} / 2$ to the centre of the vMTFs. The PDMS substrate with thickness $14.5 \mu \mathrm{m}$ (Alford et al., 2011; Shim et al., 2011) was simulated with the 20-node quadratic brick-shaped three-dimensional solid finite elements, i.e. C3D20 in ABAQUS notation, which can capture the bending deformation of the substrate. As measured in the experiment (Alford, et al., 2011), the averaged thickness of the patterned tissues was $4 \mu \mathrm{m}$ and the radius of the curvature of the deformed vMTFs was at the range of $1 \sim 10$ $\mathrm{mm}$. Hence, the bending deformation of the patterned tissues has a negligible effect on the response of the vMTFs. To simplify the calculation, the patterned tissues was simulated with 6-node quadratic triangular membrane finite elements, i.e., M3D6 in ABAQUS notation, which ignored the bending deformation of the patterned tissues. The rate constants and the material parameters for the active materials employed in the simulation were chosen as $k_{2}=k_{5}=0.5 \mathrm{~s}^{-1}, k_{3}=0.4 \mathrm{~s}^{-1}, k_{4}=0.1 \mathrm{~s}^{-1}, k_{7}=0.02 \mathrm{~s}^{-1}, k_{10}=0.3 \mathrm{~s}^{-1}, \dot{\varepsilon}_{o}=0.03 \mathrm{~s}^{-1}, \sigma_{\max }=180 \mathrm{kPa},[\eta]_{\max }=0.8$. The neo-Hookean parameters for passive behaviour of a cell include initial bulk modulus $\bar{\kappa}_{c}=3.33 \mathrm{kPa}$ and the initial elastic modulus $\bar{E}_{c}=2.0 \mathrm{kPa}$. These parameters were estimated from existing literature on contractility of smooth muscle cells (McGarry et al., 2009; Obbink-Huizer et al., 2014; Hai and Murphy, 1988b; Alford, et al., 2010). The PDMS substrate is again treated as a neo-Hookean solid, see Supplementary Data for the description of the constitutive model. The bulk modulus and the initial elastic modulus of the substrate employed in the simulation were estimated as $\bar{\kappa}_{s}=25 \mathrm{MPa}$ and $\bar{E}_{s}=1.5 \mathrm{MPa}$, respectively (Shim et al., 2011). The focal adhesion between the micropatterned tissues and the PDMS substrate was simulated with the contact model described in Supplementary Data with focal adhesion stiffness $k_{v}=k_{o} \psi=500 \mathrm{nN} \mu \mathrm{m}^{-3}$, where $k_{o}$ represents the effective spring constant of a single ligand-receptor bond, $k_{o} \sim 1 \mathrm{nN} \mu \mathrm{m}^{-1}$ (Dembo, 1994), and $\psi$ the concentration of ligand-receptor bonds per unit cell membrane area. The value of $\psi$ is estimated based on the numerical studies reported by McGarry et al. (2009) and Pathak et al.(2008), i.e. $\psi=3333 \mu \mathrm{m}^{-2}$.Numerical studies showed that the simulation results were not 
sensitive to $k_{o}$ when $k_{o}=0.015 \sim 1.5 \mathrm{nN}_{\mu \mathrm{m}^{-1}}$. This is consistent with experimental measurements that demonstrate the contractile response of vMTFs was not very sensitive to two types of extracellular matrices with different contractile phenotypes, i.e. laminin and fibronectin (Alford, et al., 2011). In the experimental study reported by Alford, et al. (2011), the micropatterned vMTFs was stimulated with the vasoconstrictor endothelin-1 (ET-1) (50 nM) and followed by the treatment of $100 \mu \mathrm{M}$ HA-1077, a rho-associated kinase (ROCK) inhibitor that was sufficient to inhibit contraction of the vMTFs. ET-1 triggers transient increase in intracellular $\mathrm{Ca}^{2+}$ level (Emori, et al., 1991) and HA-1077 inhibits Rho-kinase dependent contraction of stress fibres (Katoh, et al., 2011). In this paper, the effects of ET-1 and HA-1077 will be reflected through the time histories of $k_{1}$ and $k_{6}$. However, as the detailed information on the time history responses of the micropatterned vMTFs was not presented in the article, $k_{1}$ and $k_{6}$ cannot be calibrated based on the experimental results provided in Alford, et al. (2011). The same group has reported the detailed experimental results on the response of the vMTFs with monolayered anisotropic tissues subjected to the same time histories of soluble factors (Alford, et al., 2010). The experimental results were used to calibrate the time histories of $k_{1}$ and $k_{6}$. The finite element model for the monolayered vMTFs is shown in Fig. 3(b). The rate constants and material parameters as well as the details of the FE modelling employed in the simulation were the same as those for vMTFs with micropatterned tissues, as described before. Figure 4 (a) shows the calibrated time histories of $k_{1}$ and $k_{6}$ employed in the simulation, which can be described in the following equations

$$
k_{1}=k_{6}=\left\{\begin{array}{cc}
0.096 & \mathrm{t} \leq 0 \mathrm{~s} \\
0.018\left[1.0-\exp \left(-\frac{t+1000}{500}\right)\right]\left[0.99 \exp \left(-\frac{t+1000}{5200}\right)+0.01\right] & 0 \mathrm{~s} \leq \mathrm{t} \leq 1200 \mathrm{~s} \\
0.0048\left[1.0-\exp \left(-\frac{t-1200}{10}\right)\right]\left[0.99 \exp \left(-\frac{t-1200}{2000}\right)+0.01\right] & 1200 \mathrm{~s} \leq \mathrm{t} \leq 3000 \mathrm{~s}
\end{array}\right.
$$

where the initial values of $k_{1}$ and $k_{6}(t \leq 0)$ are defined to trigger basal response that may depend on the initial condition of the cells such as the condition for cell seeding and culture. This reflects the scenarios that the basal response of cells/tissues is triggered by calcium influx through channels in the cell membrane and $\mathrm{Ca}^{2+}$ released from intracellular stores (Jackson, 2000); the values for $0 \mathrm{~s} \leq \mathrm{t} \leq 1200 \mathrm{~s}$ are defined for the rise of intracellular $\mathrm{Ca}^{2+}$ concentration induced by ET-1; the values for $1200 \mathrm{~s} \leq \mathrm{t} \leq 3000 \mathrm{~s}$ are defined for decline of the $\mathrm{Ca}^{2+}$ concentration induced by HA-1077. In Eq. (14), the effect of each soluble factor, i.e. ET-1 and Rock inhibitor HA-1077, is modelled by multiplication of two natural exponential functions with the first natural exponential function dictating the initial 
increase of the triggered signal and second natural exponential function dictating the decay of the signal as time increase. The calibration was the process of evaluating and adjusting the values of $k_{1}$ and $k_{6}$ to fit the experimental measurement by Alford, et al.(2010). The response of the vMTFs with monolayered anisotropic tissues is shown in Fig. 4(b) and (c) for time histories of the radius of curvature and maximum principal stress at the centre of the tissue, respectively. Owing to edge effect at $\mathrm{X}=0$, the curvature is not constant along the $\mathrm{x}$-direction: there is slight difference at $\mathrm{X}=0$ and $\mathrm{X}=\mathrm{L} / 2$. The results shown in the Figs. 4 (b) were taken from $\mathrm{X}=\mathrm{L} / 2$. The FE simulation results show that the magnitude of the maximum principal stress at any point inside the tissue is nearly the same. The rate constants $k_{8}$ and $k_{9}$ relate to the strain rate effect of isotonic contraction. To examine their effects, three selected values of $k_{8}$ and $k_{9}$ were used in the simulation, i.e. $k_{8}=k_{9}=1,2,3 \mathrm{~s}^{-1}$. However, as the strain of the tissues is small, the response is essentially isometric. Hence, the response of vMTFs is not very sensitive to $k_{8}$ and $k_{9}$. The time history of radius of curvature predicted by the FE simulation agrees well with that obtained by the experimental measurement. The stress in the tissues predicted by a semi-analytical model reported in Alford, et al. (2010) is also shown in Fig. 4 (c) for comparison. The agreement again is reasonably good. The simulation results of the vMTFs with micropatterned tissues are presented next based on the FE model and the rate / material parameters described in the section.

\section{Results and discussion}

In the experiment reported by Alford et al. (2011), micro-tissues were constructed as 20, 40,60, 80 and 100 mm wide lines separated by $100 \mathrm{~mm}$ gaps within the vMTFs. To examine the effect of the width of the micropatterned tissues within the vMTFs, the numerical simulation was conducted for the micropatterned vMTFs with tissue width $\mathrm{b}=$ $20,40,60,80,100 \mu \mathrm{m}$ and $\mathrm{d}=100 \mu \mathrm{m}$ (Fig.3a). Figures $5(a)$ and $(b)$ show the time histories of the radius of curvature of the vMTFs and maximum principal stress at the centre of the micropatterned tissues, respectively. I will later show that the magnitude of the maximum principal stress at any point within a micropatterned tissue is nearly identical at a time instant. As the tissue response is essentially isometric, the time history of the maximum principal stress of the micropatterned tissues is not sensitive to the tissue width $b$ and is dictated by Eq. (10). The radius of curvature of vMTFs is sensitive to the tissue width $b$, i.e. the radius of curvature increases as the tissue width $b$ decreases. This may be ascribed to different tissue (active material) area fractions within these vMTFs, which is defined as surface area covered by the micro-tissues divided by the surface area of the vMTFs. 
Alford et al. (2011) has calculated the stress in the micropatterned tissues based on the measured curvature of vMTFs using the semi-analytical model described in Alford et al. (2010). The results for basal response and contraction stress induced by $50 \mathrm{nM}$ ET-1 are compared with those obtained by the FE simulation in Fig.5 (c). There is a certain discrepancy between the two calculations for basal response. The basal response of cells/tissues could be triggered by calcium influx through channels in the cell membrane and $\mathrm{Ca}^{2+}$ released from intracellular stores (Jackson, 2000). These may relate to the initial condition of the cells/ tissues such as the conditions of cell seeding and culture employed in the experiment. It is worth noting that the rate constants $k_{1}$ and $k_{6}$ were calibrated against the experimental measurements for the monolayered anisotropic tissues (Fig. 4a; Alford, et al., 2010), which adopted the experimental protocol of cell seeding and culture different from that of the micropatterned tissues (Alford et al., 2011). This might be the main reason for the discrepancy of the two basal response calculations. In addition, the stronger contractile output of the cells in the tissue with $20 \mu \mathrm{m}$ width patterning may play a certain role in the discrepancy.

The $50 \mathrm{nM}$ ET-1 induced contraction stress in micropatterned tissues should mainly be governed by the characteristics of the cytoskeletal contractility of the cells inside the tissues rather than the initial condition of the cells/ tissues. Good agreement has been achieved for the predictions of ET-1 induced contraction stress between the numerical simulation and the results of Alford et al. (2011) for the tissues with 40, 60, 80 and $100 \mu \mathrm{m}$ width patterns. This may suggest the contraction stress is weakly sensitive to the tissue width for these patterns. However, for $20 \mu \mathrm{m}$ width tissue patterning, the numerical simulation result is less than the average value of experimental measurements, which may suggest the thinner and more elongated spindle-like cells within the $20 \mu \mathrm{m}$ width tissue patterning have higher contractile output.

At any point within the micro-tissues, the fraction of attached myosin molecules $\eta$ is a function of orientation $\theta$. To examine the evolution of the stress fibres, Fig. 6 shows the orientation of the maximum attached myosin molecules $\eta_{\max }=\max [\eta(\theta)]$ within the tissues of the micropatterned vMTFs with $\mathrm{d}=100 \mu \mathrm{m}$ and $\mathrm{b}=100 \mu \mathrm{m}$ at selected time instants. The orientation of $\eta_{\max }$ has been found to be nearly identical to the orientation of the maximum principal stress. For the microtissues close to the centre of the vMTFs $(X=L / 2)$, the orientation of $\eta_{\max }$ is aligned with the longitudinal direction ( $\mathrm{Y}$-axis direction) of the microtissues for basal response $(\mathrm{t}=0 \mathrm{~s})$ and under the effect of ET-1 $(\mathrm{t}=390 \mathrm{~s}$ and $\mathrm{t}=887 \mathrm{~s})$, and evolves to the transverse direction (X-axis direction) when subjected to HA-1077 
$(t>1200 \mathrm{~s})$. Experimental measurements showed that both f-actin and nuclei were highly aligned with the longitudinal direction of the microtissues, see Fig.4 of Alford et al. (2011). However, the predicted orientation of $\eta_{\max }$ or the maximum principal stress is not always aligned with f-actin and nuclei, especially when the ROCK inhibitor HA-1077 was applied. This is consistent with the experimental measurement shown in Fig. 1 of Deshpande, et al. (2007) for a cell resting an array of micro-posts, which demonstrates that there is little correlation between the orientation of the f-actin fibres and the direction of the force vectors. For the tissues close to free end $(\mathrm{X}=0)$, the stress fibres within the tissues are less aligned owing to the edge effect. In Fig.6, the colour of the arrows, which indicate the orientation of $\eta_{\max }$, is related to the value of the maximum principal stress within the microtissues. As the response is essentially isometric, the maximum principal stress at any point within a microtissue is nearly identical at a time instant.

VMTFs can potentially be used to study the mechanics of SMCs based tissues, e.g. mimicking the lamellae of the arterial tunica media (Alford et al., 2011). As mentioned by Murphy (1988), cells can be arranged in series as well as in parallel within a tissue and force generating capacity of the tissue is directly related to the arrangement of cells. In Supplementary Data, the simulation results for the vMTFs with micropatterned tissues in three types of arrangements are presented, which may be used for future experimental development. $\mathrm{Ca}^{2+} / \mathrm{MLCK}$ pathway was considered through rate constants $k_{1}$ and $k_{6}$ for the simulations described in the section. However, as reviewed by Katoh, et al. (2011), contraction of stress fibres can also be regulated by $\mathrm{Ca}^{2+}$ - independent Rho kinase pathway. In the model described in Eqs. (1) through (4), $\mathrm{Ca}^{2+}$ - independent Rho kinase pathway can be modelled through rate constants $k_{2}, k_{5}, k_{3}$ and $k_{4}$ (Liu, 2014).To understand the effects of these rate constants , parameter study has been conducted via the numerical simulations of monolayered vMTFs, as shown in Supplementary Data. It is demonstrated that the numerical results are sensitive to these rate constants. Further research will be developed to model $\mathrm{Ca}^{2+}$ - independent Rho kinase pathway via rate constants $k_{2}, k_{5}, k_{3}$ and $k_{4}$.

\section{Simulation of endothelial cells on an array of micro-posts}

To examine the effects of vasoactive soluble factors on the contractility of endothelial cells, Yang, et al. (2011) experimentally investigated the mechanical response of single endothelial cells resting on an array of micro-posts. The deflections of the micro-posts were measured under the conditions that the cells were subjected to a range of 
soluble factors such as Lysophosphatidic acid (LPA), vascular endothelial growth factor (VEGF), bovine serum albumin (BSA), Histamine, Thrombin, sphingosine-1-phosphate (S1P) and serum. The micro-posts were hexagonally arranged in two-dimensional space with centre-to-centre spacing $l_{1}=4 \mu \mathrm{m}$. Each micro-post had a diameter of $d_{1}=$ $1.83 \mu \mathrm{m}$, a height of $h=8.3 \mu \mathrm{m}$, and Young's modulus of $E=2.55 \mathrm{kPa}$. Hence, the bending stiffness of a micro-post can be calculated as $k_{n}=3 \pi E d_{1}^{4} / 64 h^{3}=7.22 \mathrm{nN} \mu \mathrm{m}^{-1}$. Multiplying the deflections of the micro-posts by the bending stiffness and integrating over the magnitudes of the deflections, the strain energy and contractile work generated by a cell can be quantified in the experiment. In this section, the endothelial cell-substrate dynamic interaction event is simulated. The aims of the simulation are to (i) examine the ability of the non-smooth muscle type constitutive model, described in Section 2, in prediction of contractile response of the non-SMCs based on the calibrated rate constants, (ii) capture the observed strain energy density distribution within the cell during the cell-substrate interaction event, and (iii) investigate the effect of the effective stiffness and Possion's ratio of the substrate on cell polarity.

\section{The finite element model}

The deformation of the cells during the experiment was dominated by in-plane deformation. Hence, the cells were simulated with a 6-node quadratic triangular membrane finite element, i.e. M3D6 in ABAQUS notation. The rate constants and the active material parameters employed in the non-SMCs constitutive model were chosen as $k_{2}=0.5$ $\mathrm{s}^{-1}, k_{4}=0.1 \mathrm{~s}^{-1}, k_{8}=3 \mathrm{~s}^{-1}, k_{10}=0.3 \mathrm{~s}^{-1}, \dot{\varepsilon}_{o}=0.03 \mathrm{~s}^{-1}, \sigma_{\max }=16 \mathrm{kPa}$ and $[\eta]_{\max }=0.8$, The neo-Hookean parameters for passive behaviour of a cell were chosen as initial bulk modulus $\kappa=0.333 \mathrm{kPa}$ and Young's modulus $E=0.2 \mathrm{kPa}$. These parameters were estimated from McGarry et al. (2009) and Shukla et al. (2004). To simplify the simulation, the micro-post array based substrate was treated as a three-dimensional continuum with effective Young's modulus $E_{1}=11.6 \mathrm{nN} \mu \mathrm{m}^{-2}$ and effective Poisson's ratio $v_{1}=0$, and was simulated with a 20-node quadratic brick-shaped three-dimensional solid finite element, i.e. C3D20 in ABAQUS notation. Similar to the simulation of vMTFs in Section 3, the simplified model for focal adhesion as described in Supplementary Data was employed to simulate the adhesion of cells and the underlying substrate with focal adhesion stiffness $k_{v}=k_{o} \psi=500 \mathrm{nN} \mu \mathrm{m}^{-3}$. Numerical study has demonstrated that the simulation results are not sensitive to the value of $k_{o}$ when $k_{o}$ is within a practical range, i.e. $k_{o} \sim 1 \mathrm{nN} \mu \mathrm{m}^{-1}$ (Dembo, 1994). However, the results become sensitive when $k_{o} \sim 0.1-0.01 \mathrm{nN} \mu \mathrm{m}^{-1}$. 
The time histories of the rate constants $k_{1}$ and $k_{3}$ depend on the effects of soluble factors. As reported by Yang et al. (2011), soluble factors, such as Lysophosphatidic acid (LPA), S1P, thrombin and serum, promote phosphorylation of myosin light chain II for endothelial cells .Hence, the effects of these soluble factors can be reflected through $k_{1}$ in the constitutive model. However, some soluble factors, such as Histamine and vascular endothelial growth factor (VEGF), cause significant increases in actin polymerization but have a small effect on phosphorylation of myosin light chain II. This basically increases the number of myosin-binding sites on actin filaments and, therefore, increases the attachment rate of myosin to actin filaments. Hence, the effects of Histamine and VEGF can be reflected through the values of $k_{3}$. The simulations described next is based on the calibrated value of $k_{1}$ for the effect of LPA. The simulations based on the calibrated value of $k_{3}$ for the effect of VEGF are described in Supplementary Data.

The time history of $k_{1}$ employed in the simulation for LPA $\left(10 \mu \mathrm{g} \mathrm{mL}^{-1}\right)$ was obtained through calibration against measured time history of the strain energy stored in the cell, which can be described in the following equations and is shown in Fig. 7 ,

$$
k_{1}=\left\{\begin{array}{cc}
0.045 & t<600 \mathrm{~s} \\
0.24\left[1-\exp \left(-\frac{t-350}{600}\right)\right]\left[0.99 \exp \left(-\frac{t}{1600}\right)+0.01\right] & 600 \mathrm{~s} \leq \mathrm{t} \leq 2400 \mathrm{~s}
\end{array}\right.
$$

In Eq. (15), the initial value of $k_{1}(t<600 \mathrm{~s})$ is defined to trigger basal response and the value for $600 \mathrm{~s} \leq \mathrm{t} \leq 2400 \mathrm{~s}$ is defined for the rise of intracellular $\mathrm{Ca}^{2+}$ concentration induced by LPA. During the calculation, the time history of $k_{3}$ is kept at the basal level, namely, $\mathrm{k}_{3}=0.2 \mathrm{~s}^{-1}$. In Eq. (15), the effect of each soluble factor, i.e. LPA, is again modelled by multiplication of two natural exponential functions with the first natural exponential function dictating the initial increase of the triggered signal and second natural exponential function dictating the decay of the signal as time increase. Based on Eq. (15), the time history of strain energy of the cell obtained by the numerical simulation agrees well with that obtained by experimental measurement, as shown in Fig.7. The rate constants $\mathrm{k}_{8}$ relates to the strain rate effect of isotonic contraction of the cell. To examine its effect, three selected values of $\mathrm{k}_{8}$ were used in the simulation, i.e. $\mathrm{k}_{8}=0.3,3,30 \mathrm{~s}^{-1}$. As shown in Fig.7, the simulation results are not very sensitive to the strain rate 
within the cell for $\mathrm{k}_{8}=0.3 \sim 3 \mathrm{~s}^{-1}$; the results become sensitive when $\mathrm{k}_{8}$ takes a large number, say $\mathrm{k}_{8}=30 \mathrm{~s}^{-1}$. For higher values of $\mathrm{k}_{8}$, the strain energy stored in the cell becomes smaller.

\section{Results and discussion}

Figure 8 ( $a$ and $b$ ) show the predicted and measured contours of the strain energy density stored in the cell at selected time instants. The deformation of the cell obtained by the simulation is also shown in the figure. As the micro-post array based substrate was simplified as a three-dimensional continuum in the simulation, the detailed distribution of the interfacial deformation between the cell and substrate may not be able to be predicted accurately. However, there is reasonable correlation between the predicted and measured distribution of the strain energy density within the cells. Consistent with experimental observations, the deformation of the cell diminishes from the edge to the centre and the central part of the cell is essentially under isometric state, i.e. the deformation is close to zero.

Using the numerical simulation, the effects of the effective stiffness and Possion's ratio of the substrate can be investigated with respect to triggering cell polarity. Cell polarity refers to spatial differences in the structure, shape and function of cells. Cell polarity enables cells to carry out specialized functions. Hence, it may be useful to control the cell polarity in order to understand its effect on cellular functions. Numerical simulations were conducted for an endothelial cell resting on a substrate with dual rigidity, i.e. half of the substrate with effective elastic modulus $E_{1}=11.6 \mathrm{nN} \mu \mathrm{m}^{-2}$ and the other half with doubled elastic modulus $E_{2}=23.2 \mathrm{nN} \mu \mathrm{m}^{-2}$, and all with Poisson's ratio $\nu=0$. The dual rigidity could be achieved by letting half of the substrate with height of the posts $h=8.3 \mu \mathrm{m}$ and the other half with $h=4.15 \mu \mathrm{m}$. A similar strategy has been employed by Breckenridge et al. (2014) in studying the contractile response of stem cells. The predicted contractile response of the endothelial cell is shown in Fig. 9(a) for time history of strain energy and Fig. 9 (b) for contours of displacement and strain energy density at selected time instants. Cell polarity was induced with larger deformation on the softer part of the substrate: the peak deformation is nearly two times higher than that on stiffer part of the substrate. Compared to the response of the single rigidity substrate case shown in Fig. 7, the peak strain energy stored in the cell decreased as the stiffness of half of the substrate increased by twice.

The effective Poisson's ratio could be manipulated through the arrangement of posts or controlling the microstructure of the substrate (Zhang et al., 2013). To understand its effect, numerical simulations were conducted for the singlerigidity substrate case with the effective Poisson's ratio increased to 0.45 . The corresponding results are shown in 
Fig. 9 (a) for the time history of strain energy and in Fig. 9(c) for the contours of displacement and strain energy density at selected time instants. Compared to the simulation results in Fig.7 and Fig. 9 (a), there is nearly no change for the time history of strain energy. However, the cell exhibits certain cell polarity (Fig.9 (c)).

\section{Concluding remarks}

Numerical simulations of contractile responses of micropatterned vascular muscular thin films (vMTFs) and endothelial cells resting on an array of micro-posts under stimulation of soluble factors have been conducted based on the bio-chemo-mechanical constitutive models for cytoskeletal contractility. Two types of constitutive models have been employed in the simulations, i.e. smooth muscle cells and non-smooth muscle cells. The time histories of the effects of soluble factors have been obtained via calibrating rate constants ( $k_{1}$ and $k_{6}$ ) against experimental measurements of contractile responses of tissues or cells. The initial values of $k_{1}$ and $k_{6}(t \leq 0)$ have been defined to trigger the basal responses. In the experiment reported by Alford et al. (2011), micro-tissues were constructed as 20, 40,60, 80 and $100 \mathrm{~mm}$ wide lines separated by $100 \mathrm{~mm}$ gaps within the vMTFs. The numerical parameter study for the vMTFs suggests the overall contractile response of the micro-tissues is essentially isometric, i.e. the strain rate effect is negligible. Hence, the predicted time history of the maximum principal stress of the micropatterned tissues is not sensitive to the micro-tissue width $b$ and is dictated by Eq. (10). The numerical results suggest that the radius of curvature of vMTFs is sensitive to the micro-tissue width $b$, i.e. the radius of curvature increases as the micro-tissue width $b$ decreases. This may be ascribed to different tissue (active material) area fractions within these vMTFs, which is defined as surface area covered by the micro-tissues divided by the surface area of the vMTFs. Good agreement has been achieved for predictions of ET-1 induced contraction stress between the FE numerical simulation and the experimental results of Alford, et al. (2011) for the tissues with 40, 60, 80 and $100 \mu \mathrm{m}$ width patterns. This may suggest the contraction stress is weakly sensitive to the tissue width for these patterns. However, for $20 \mu \mathrm{m}$ width tissue patterning, the numerical simulation result is less than the average value of experimental measurements, which may suggest the thinner and more elongated spindle-like cells within the $20 \mu \mathrm{m}$ width tissue patterning have higher contractile output. As shown in Eq.(10), isometric stress $\sigma_{o}$ can be related to the ratio of the maximal tension and the maximal fraction of the attached myosin molecules permitted by biochemistry, $\sigma_{\max } /[\eta]_{\max }$, as well as the fraction of the attached myosin molecules $\eta$. Hence, when under an identical bio-chemical stimulation, 
elongated spindle-like cells may have either higher value of $\sigma_{\max } /[\eta]_{\max }$ or higher value of $\eta$ compared to the cells with normal cell shape . Experimental measurements (Alford, et al., 2011) showed that the both f-actin and nuclei were highly aligned with the longitudinal direction of the micropatterned tissues. At any point within the microtissues, the fraction of attached myosin molecules $\eta$ is a function of orientation $\theta$. The predicted orientation of the maximum attached myosin molecules $\eta_{\max }=\max [\eta(\theta)]$ is not always aligned with f-actin and nuclei, especially subjected to an inhibitor. The orientation of $\eta_{\max }$ has been found to be nearly identical to the orientation of the maximum principal stress. This is consistent with the experimental measurements shown in Fig. 1 of Deshpande, et al. (2007) for a cell resting an array of micro-posts, which demonstrates there is little correlation between the orientation of the f-actin fibres and the direction of the force vectors.

The constitutive model for non-smooth muscle cells was used to simulate the contractile response of the endothelial cells resting on an array of micro-posts. The time histories of $k_{1}$ and $k_{3}$ were calibrated to reflect the effects of Lysophosphatidic acid (LPA) and vascular endothelial growth factor (VEGF), respectively. The numerical simulation shows that the deformation of the cell diminishes from edge to centre and the central part of the cell is essentially under isometric state. Using numerical simulations, the effects of the effective stiffness and Possion's ratio of the substrate were investigated with respect to triggering cell polarity. It demonstrated that cell polarity could be triggered by using dual stiffness substrate and manipulating the effective Possion's ratio of the substrate.

The simulations presented in the paper do not intend to critically examine the experimental studies reported by Alford, et al. (2011) and Yang et al. (2011) as the computational models reported in this paper are subjected to the following limitations

- The passive behaviour of a cell is treated as a neo-Hookean like solid. This may oversimplify the complexity of the passive behaviour of a cell.

- The detailed modelling of cell-cell contact as well as cell shape are not included. Hence, it could not model cell shape dependent contraction.

- As reviewed by Katoh, et al. (2011), contraction of stress fibres can be regulated by $\mathrm{Ca}^{2+} / \mathrm{MLCK}$ pathway as well as $\mathrm{Ca}^{2+}$ - independent Rho kinase pathway. The models focus on $\mathrm{Ca}^{2+} / \mathrm{MLCK}$ pathway. $\mathrm{Ca}^{2+}-$ independent Rho kinase mediated contraction of stress fibres is not explicitly modelled in the paper. 
- The detailed modelling on the network of signalling pathways that regulate the myosin light chains through both phosphorylation and dephosphorylation is not included.

The computational models can simulate the detailed responses of tissue/cell-substrate interactions under soluble factors. This can be shown in Figs.6, 8 and 9(b and c) for the evolution of stress fibre orientation or contours of strain energy density and displacement at selected time instants. Available simplified predicative models, such as the model employed in (Alford et al., 2010), may focus on predication of the overall behaviour rather than detailed responses. Further development of the computational models will address the limitations list above.

\section{Acknowledgments}

The author is grateful for access to the University of Nottingham High Performance computing facility. The work reported in the paper is supported by Tsinghua/Nottingham Research and Teaching Fund. The constructive comments from the anonymous referee are gratefully acknowledged. 


\section{References}

Alford PW, Nesmith AP, Seywerd JN, Grosberg A, Parker KK. 2011 Vascular smooth muscle contractility depends on cell shape. Integr. Biol. 3, 1063-1070.

Alford PW, Nesmith AP, Seywerd JN, Grosberg A, Parker KK. 2010 Biohybrid thin films for measuring contractility in engineered cardiovascular muscle. Biomaterials. 31, 3613-3621.

Barany M. 1967 ATPase activity of mysion correlated with speed of muscle shortening. J. Gen. Physiol. 50,197-218

Breckenridge MT, Desai RA, Yang MT, Fu J, Chen CS, 2014 Substrates with Engineered Step Changes in Rigidity Induce Traction Force Polarity and Durotaxis. Cellular and Molecular Bioengineering, 7, 26-34.

Dembo M. 1994 On peeling an adherent cell from a surface. In Vol. 24 of series : Lectures on mathematical problem in Biology, American Mathematical Society, Providence , RI ,51-77

Deshpande VS, McMeeking RM, Evans AG. 2006 A bio-chemo-mechanical model for cell contractility. Proc. Natl Acad. Sci. USA 103, 14 015-14 020.

Deshpande VS, McMeeking RM, Evans AG. 2007 A model for the contractility of the cytoskeleton including the effect of the stress fibre formulation and dissociation. Proc.R.Soc., London, A 463 , 787-815.

Emori, T., Hirata, Y., Ohta, K., Kanno, K., Eguchi, S., Imai, T., Shichiri, M. and Marumo, F., 1991. Cellular mechanism of endothelin-1 release by angiotensin and vasopressin. Hypertension, 18(2), pp.165170.

Hai CM, Murphy RA. 1988a Cross-bridge phosphorylation and regulation of latch state in smooth muscle. J. Appl. Physiol. 254, C99-C106.

Hai CM, Murphy RA. 1988b Regulation of shortening velocity by cross-bridge phosphorylation in smooth muscle. Am.J.Physiol. 255, C86-C94.

Hill AV. 1938 The heat of shortening and the dynamic constraints of muscle. Proceedings of the Royal Society of London B 126, 136-195.

Jackson WF. 2000 Ion Channels and Vascular Tone. Hypertension 35,173-178.

Katoh, K., Kano, Y. and Noda, Y., 2011. Rho-associated kinase-dependent contraction of stress fibres and the organization of focal adhesions. Journal of The Royal Society Interface, 8(56), pp.305-311.

Liu T. 2014 A constitutive model for cytoskeletal contractility of smooth muscle cells. Proceedings of the Royal Society of London A. 470, 2164

Loosli, Y., Luginbuehl, R. and Snedeker, J.G., 2010 Cytoskeleton reorganization of spreading cells on micro-patterned islands: a functional model. Philosophical Transactions of the Royal Society of London A. 368(1920), pp.2629-2652.

McGarry P, Chen CS, Deshpande VS, McMeeking RM, Evans AG. 2009 Simulation of the contractile response of cells on an array of micro-posts. Phil. Trans. R. Soc. A 367, 3477-3497.

Murphy RA. 1988 Muscle cells of hollow organs. News Physiol. Sci. 3, 124-128.

Obbink-Huizer C, Foolen J, Oomens CWJ, Borochin M, Chen CS, Bouten CVC, Baaijens FP. 2014 Computational and experimental investigation of local stress fibre orientation in uniaxially and biaxially constrained microtissues. Biomech. Model. Mechanobiol. 13, 1053-63. 
Ogden RW. 1978 Nearly isochoric elastic deformations: application to rubberlike solids. J. Mech. Phys. Solids, 26, 37-57.

Pathak A, Deshpande VS, McMeeking RM, Evans AG. 2008 The simulation of stress fibre and focal adhesion development in cells on patterned substrates. J.R.Soc. Interface 5,507-524.

Shim J, Grosberg A, Nawroth JC, Parker KK, Bertoldi K. 2012 Modeling of cardiac muscle thin films: pre-stretch, passive and active behaviour. Journal of Biomechanics.45, 832-41.

Shukla A, Dunn AR, Moses MA, Vliet KJV. 2004 Endothelial cells as mechanical transducers: Enzymatic activity and network formation under cyclic strain. Mech. Chem. Biosyst. 1, 279-290.

Tan JL, Tien J, Pirone DM, Gray DS, Bhadriraju K, Chen CS. 2003 Cells lying on a bed of microneedles: an approach to isolate mechanical force. Proc. Natl Acad. Sci. USA 100, 1484- 1489.

Yang MT, Reich DH, Chen CS. 2011 Measurement and analysis of traction force dynamics in response to vasoactive agonists. Integr. Biol. 3, 663-674.

Zhang W, Soman P, Meggs K, Qu X, Chen S. 2013 Tuning the Poisson's ratio of biomaterials for investigating cellular response. Advanced Functional Materials, 23, 3326-3232. 


\section{Figure captions}

Figure 1 Biochemical model for non-smooth muscle cells. The three possible states of a mysion molecule include : (A) free unphosprated mysion, (B) phosprated mysion without attachment with f-actin filaments and (C) phosprated mysion attached to f-actin filaments.

Figure 2 Two-dimensional representive volume element (RVE) employed in the analysis of a two-dimentional cell.

Figure 3 Finite element models for vMTFs with (a) micropatterned tissues and (b) monolayered anisotropic tissues under a deformed configurtion .

Figure 4 Response of vMTFs with a monolayered tissue under stimulation of ET-1 for $1200 \mathrm{~s}$ followed by HA1022. (a) Time history of $k_{1}$ and $k_{6}$ employed in simulations, (b) radius of curvature as a function of time obtained by experiment and simulations with selected values of $k_{8}$ and $k_{9}$, respectively, and (c) maximum principal stress as a function of time obtained by the numerical simulations with selected values of $k_{8}$ and $k_{9}$. The prediction using the method described in Alford et al. (2010) is shown in the figure for comparison.

Figure 5 Response of vMTFs with micropatterned tissues under stimulation of ET-1 for $1200 \mathrm{~s}$ followed by HA1022. Time histories of radius of curvatures (a) and maximum principal stress (b) for selected tissue pattern widths obtained by numerical simulation. (c) The stresses corresponding to basal tone and ET-1 induced contraction obtained by the simulation are compared with the results reported in Alford et al. (2011).

Figure 6 The orientation of $\eta_{\max }$ within the tissues of the micropatterned vMTFs with $\mathrm{d}=100 \mu \mathrm{m}$ and $\mathrm{b}=100 \mu \mathrm{m}$ (Fig. 3a) at selected time instants. The colour of the arrows that indicate the orientation of $\eta_{\max }$ is related to the value of the maximum principal stress within the tissues. Owing to symmetry, only half of the vMTFs is shown in the figure. The location with $X=0$ corresponds to the free end of the vMTFs. For interpretation of the color legend in this figure, the reader is referred to the web version of this article.

Figure 7 Mechanical response of an endothelial cell resting on an array of micro-posts subjected to stimulation of LPA $\left(10 \mu \mathrm{g} \mathrm{mL}^{-1}\right)$. Time histories of $k_{1}$ employed in the numerical calculation and strain energy stored in the cell obtained by experiment (Yang, et al., 2011) and numerical simulation with $\mathrm{k}_{8}=0.3,3,30 \mathrm{~s}^{-1}$.

Figure 8 Mechanical response of an endothelial cell resting on an array of micro-posts subjected to stimulation of LPA $\left(10 \mu \mathrm{g} \mathrm{mL}^{-1}\right)$. (a) and (b) contours of strain energy density of the cell obtained by experiment (Yang, et al., 2011, reproduced by permission of The Royal Society of Chemistry) and numerical simulation, respectively, as well as contours of predicted displacement of the cell at selected time instants. Scale bars are $30 \mu \mathrm{m}$. For interpretation of the color legend in this figure, the reader is referred to the web version of this article.

Figure 9 Effect of substrate for an endothelial cell resting on an array of micro-posts subjected to stimulation of LPA $\left(10 \mu \mathrm{g} \mathrm{mL}^{-1}\right)$. (a) Time histories of predicted strain energy stored in the cell resting on a dual-rigidity substrate (Poisson's ratio 0.0 ) and a single-rigidity substrate with Poisson's ratio 0.45 , respectively ; (b) and (c) contours of predicted displacement and strain energy density of the cell resting on the dual-rigidity substrate and the singlerigidity substrate with Poisson's ratio 0.45 , respectively, at selected time instants. For interpretation of the color legend in this figure, the reader is referred to the web version of this article. 


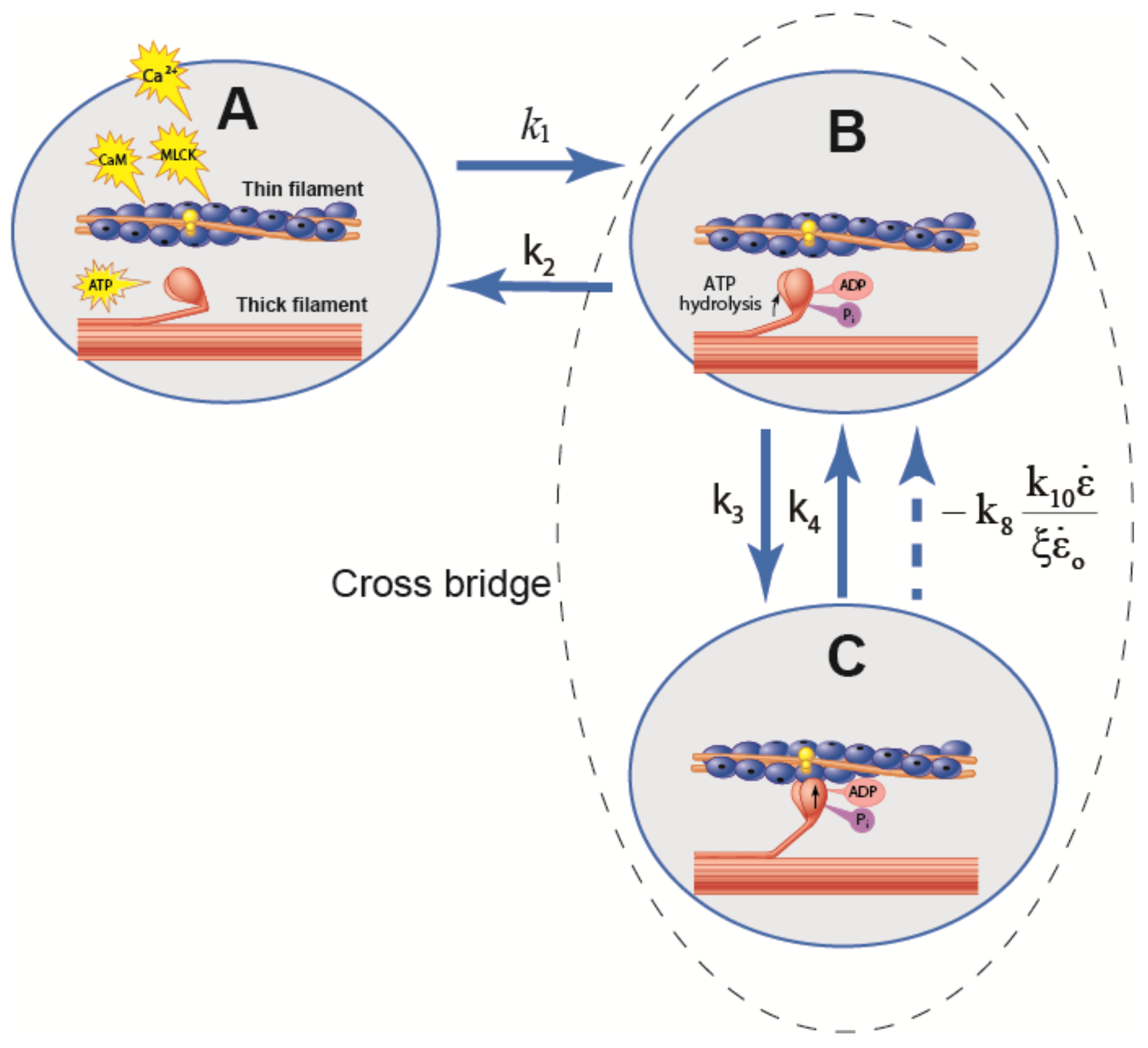

Figure 1 


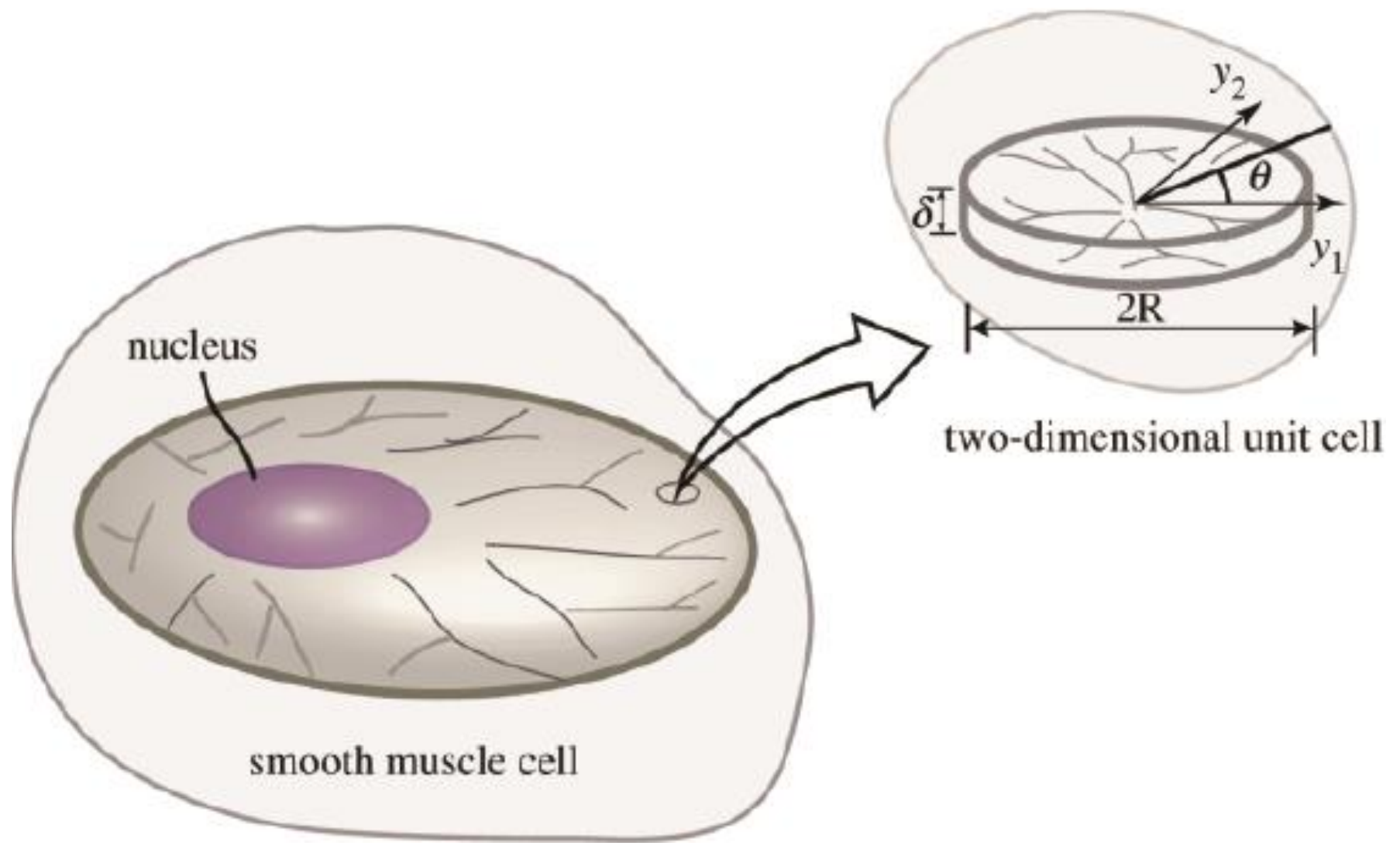

Figure 2 


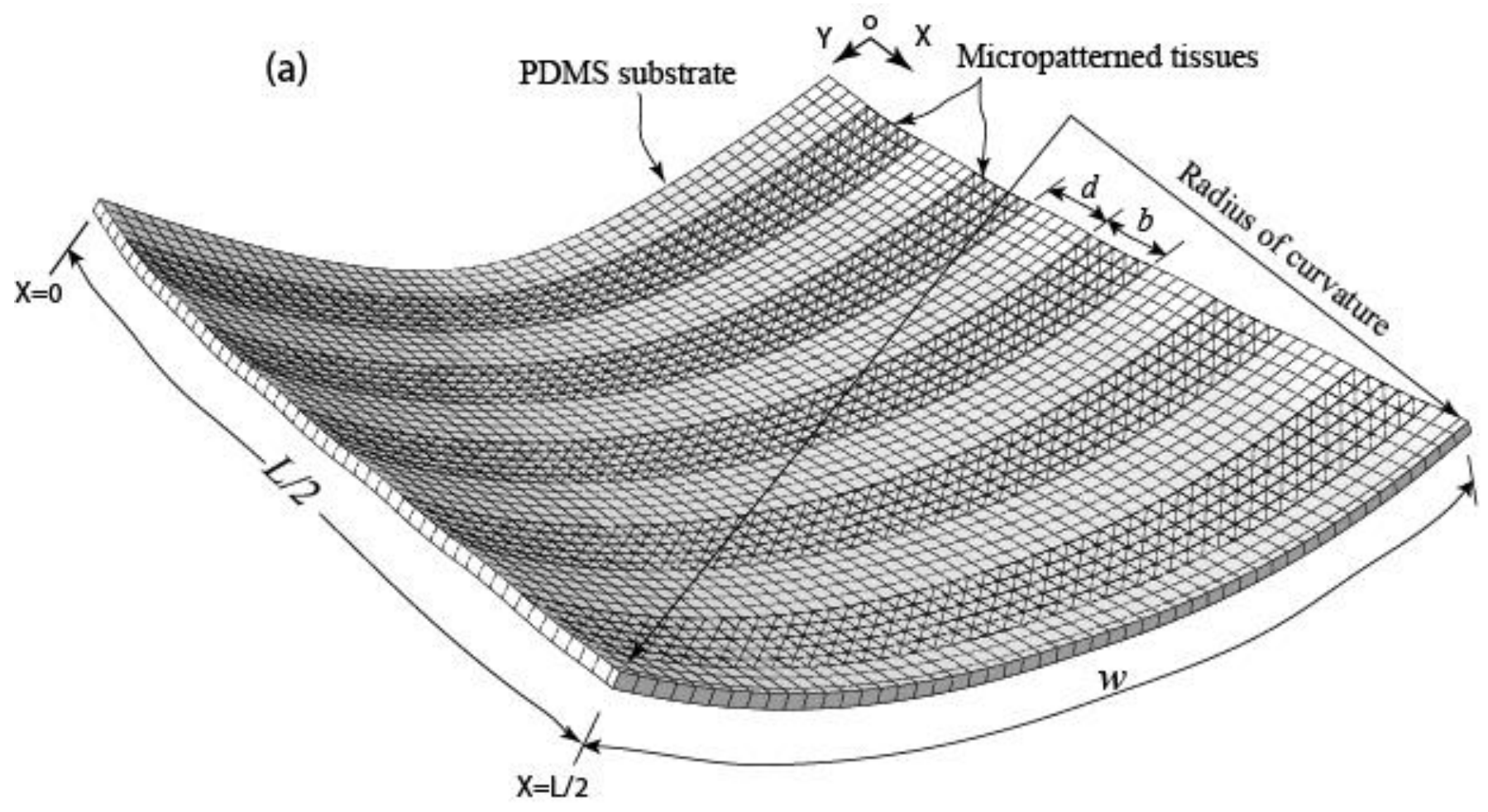

(b)

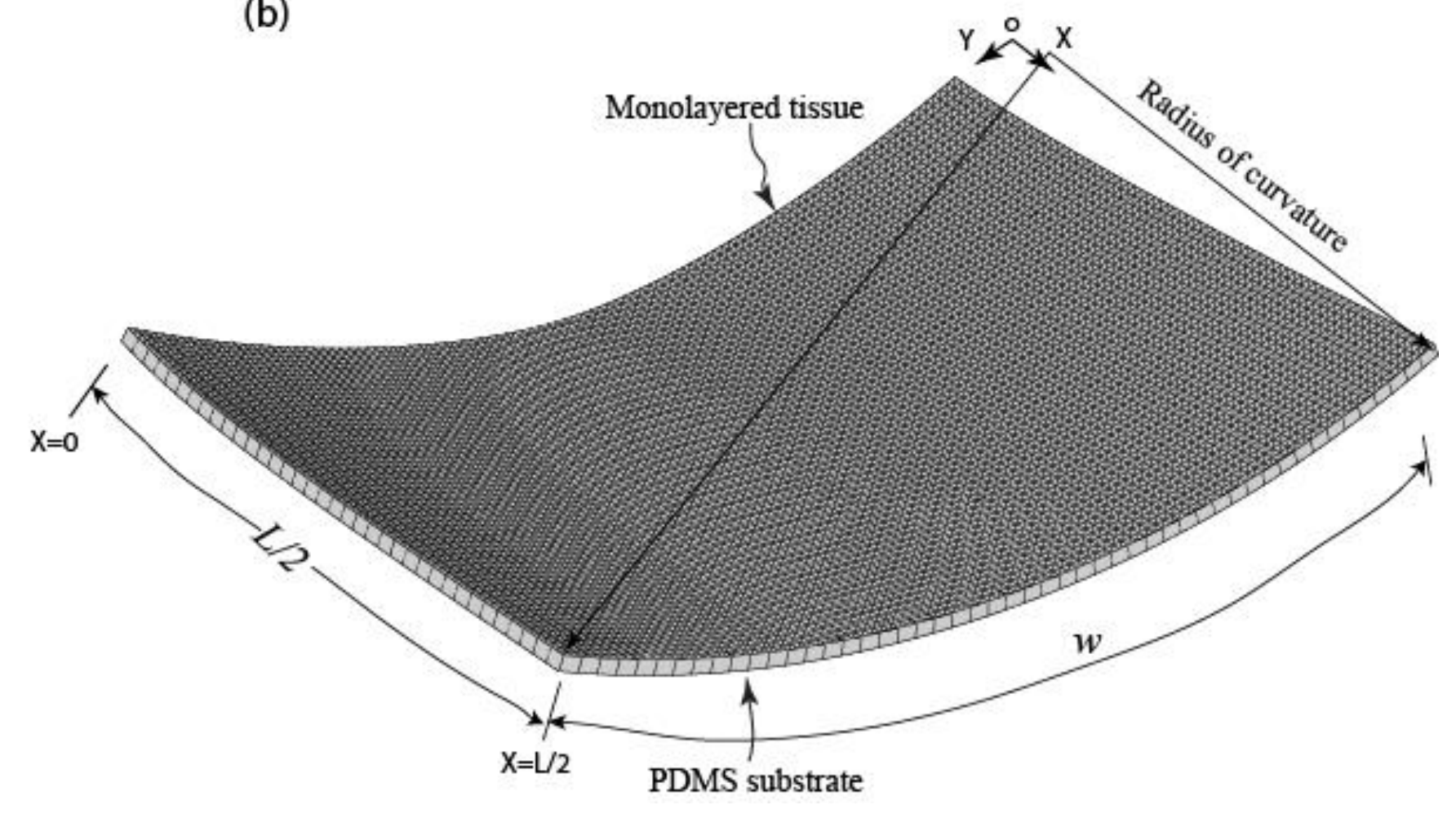

Figure 3 


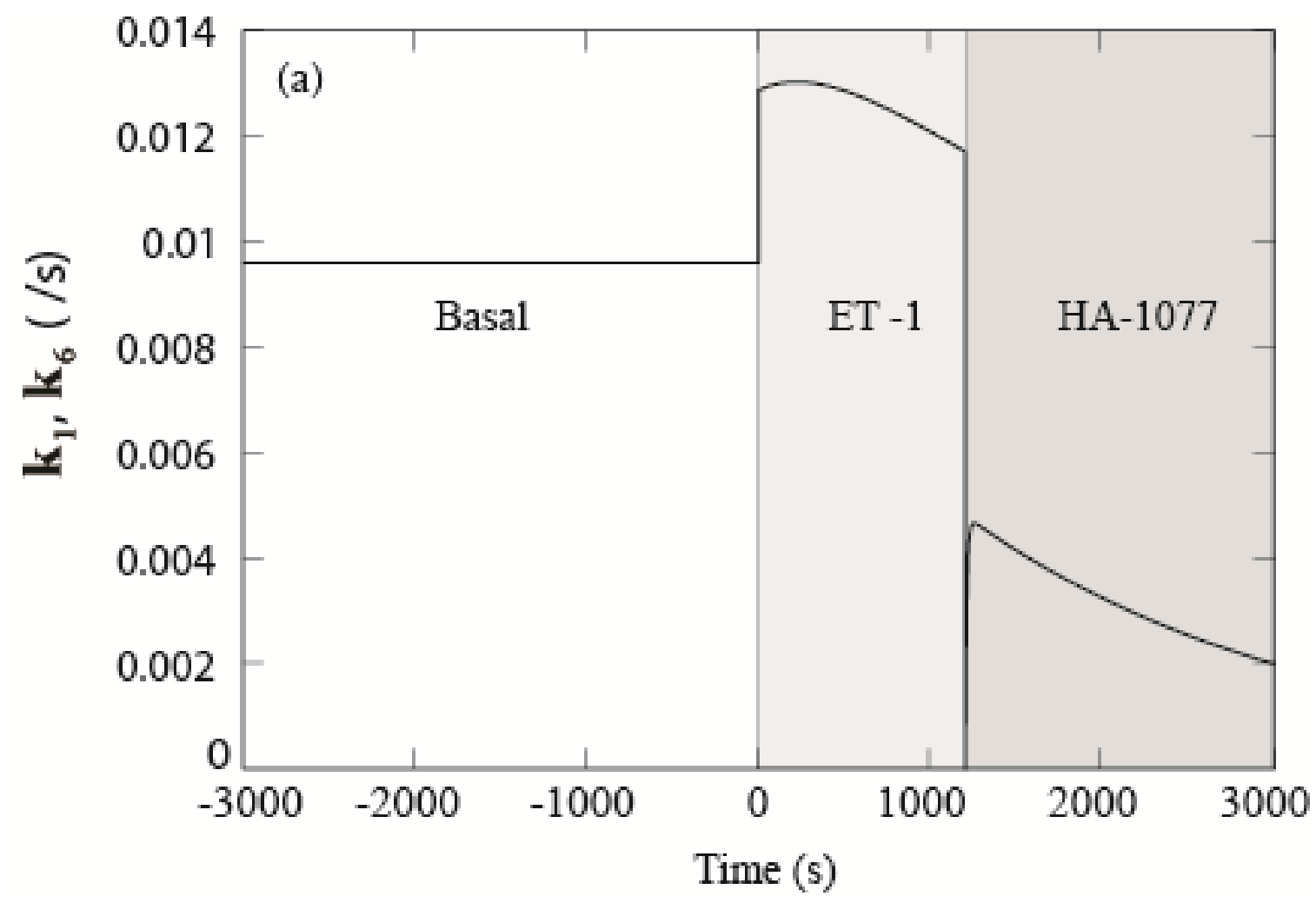



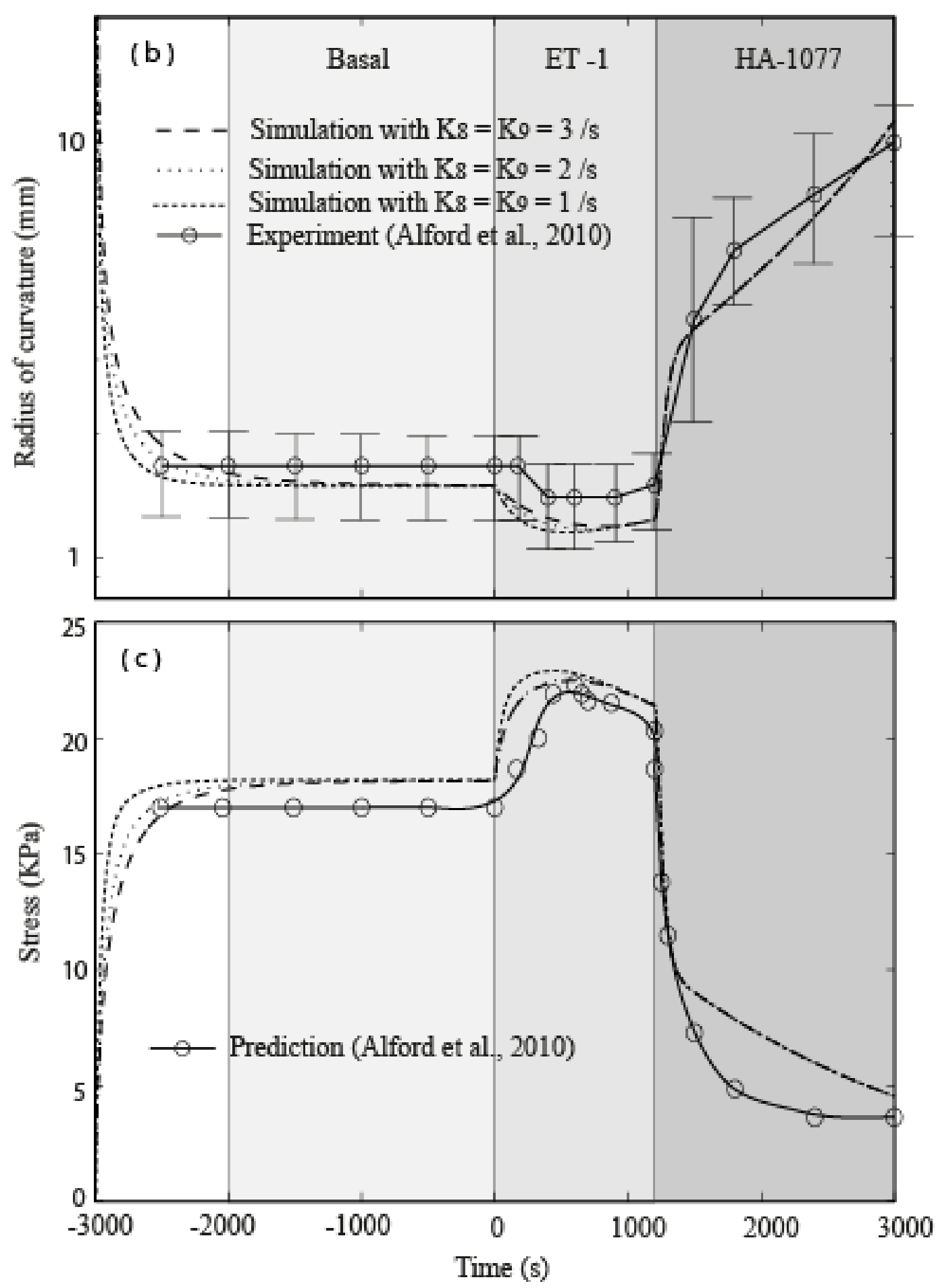

Figure 4 

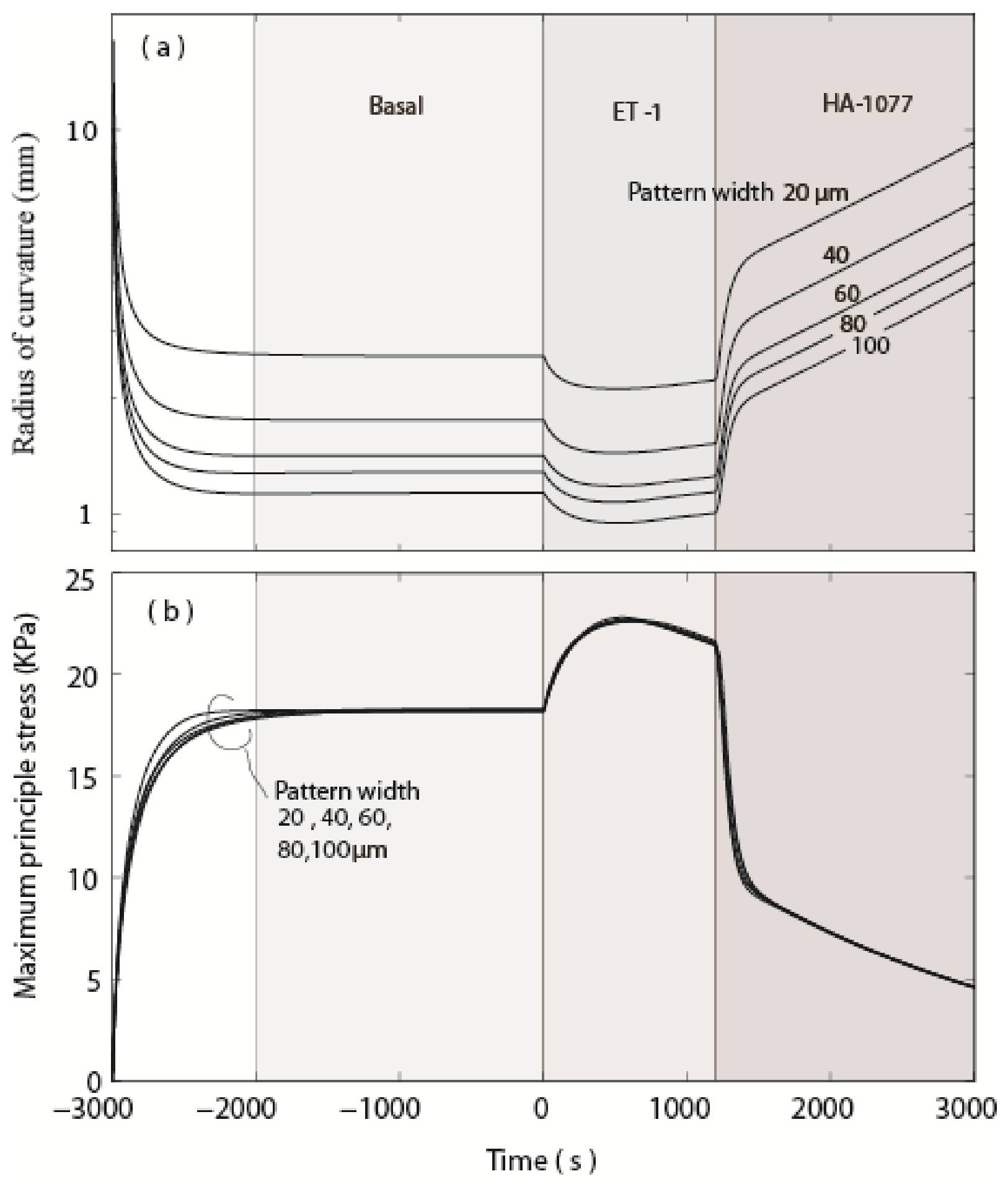


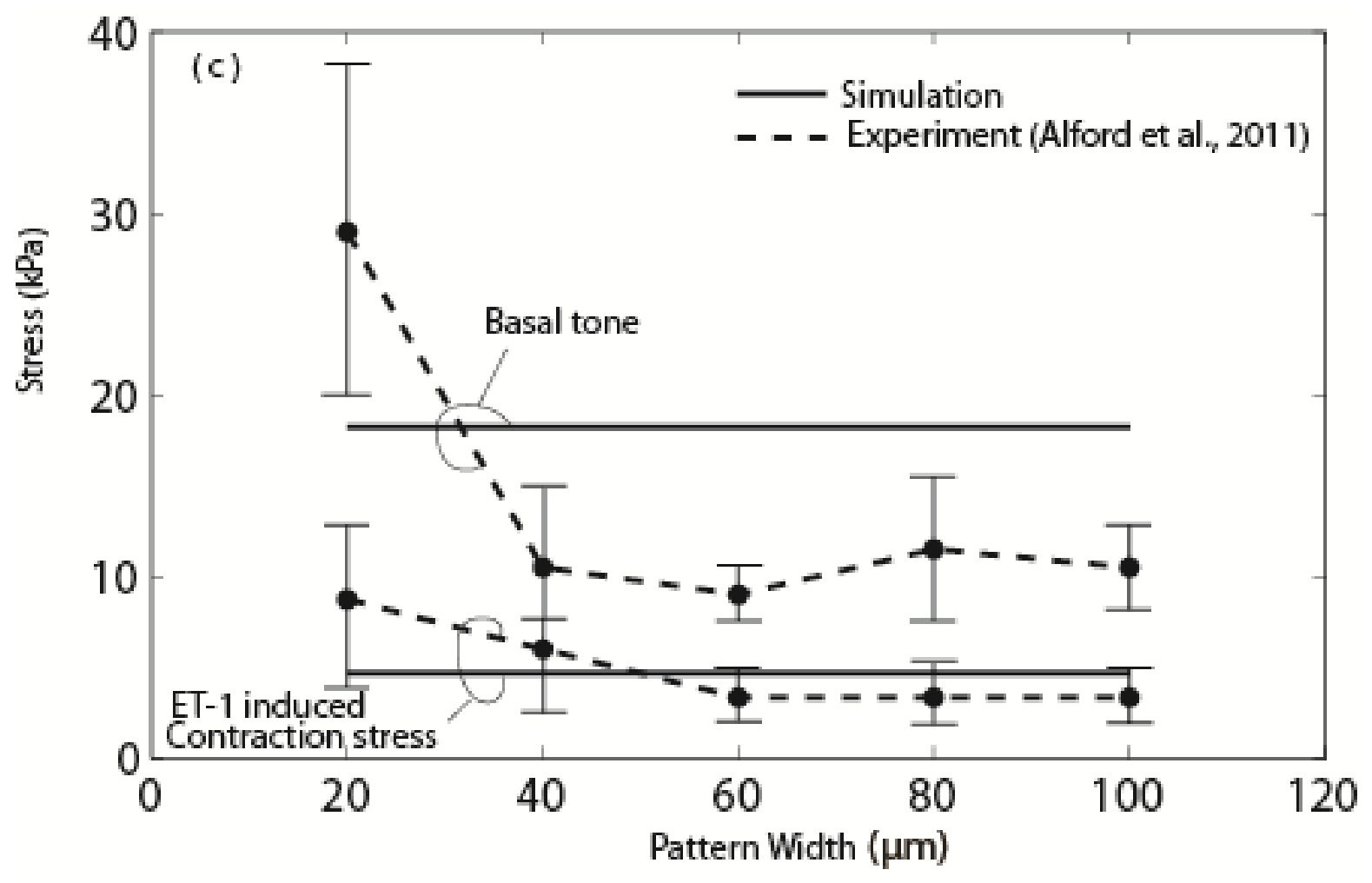

Figure 5 


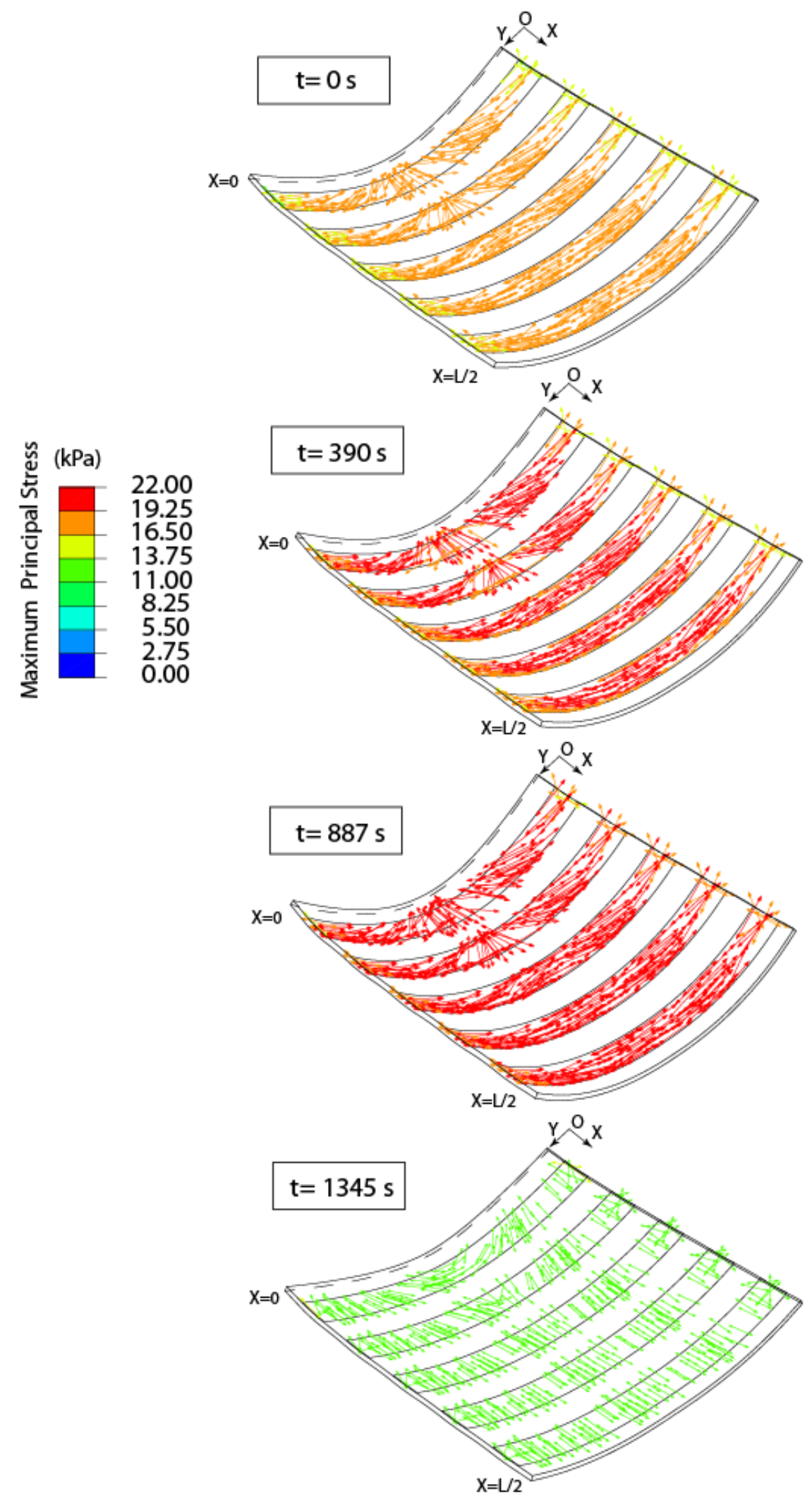

Figure 6 


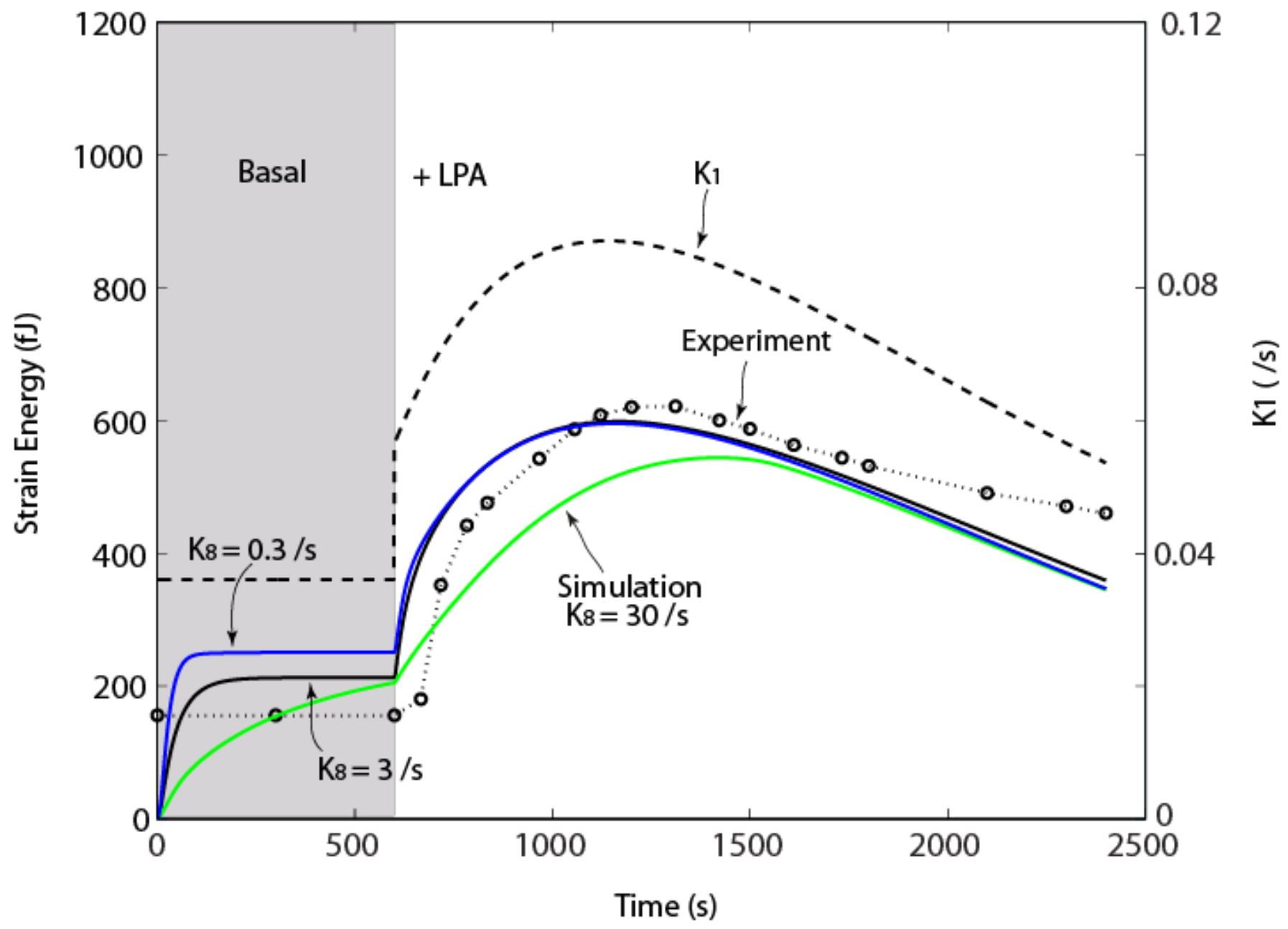

Figure 7 
(a) $t=600 \mathrm{~s}$

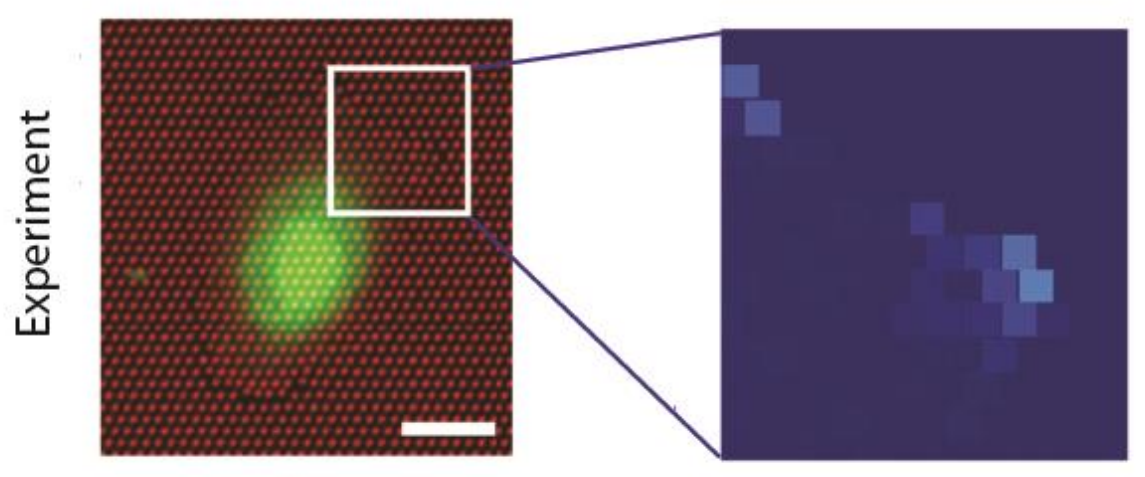

Strain Energy Density (fJ/um ${ }^{3}$ )

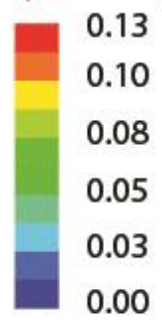

Displacement

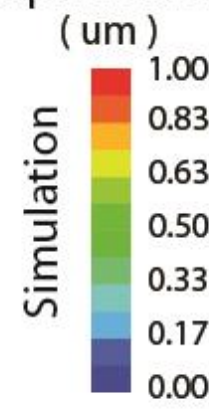

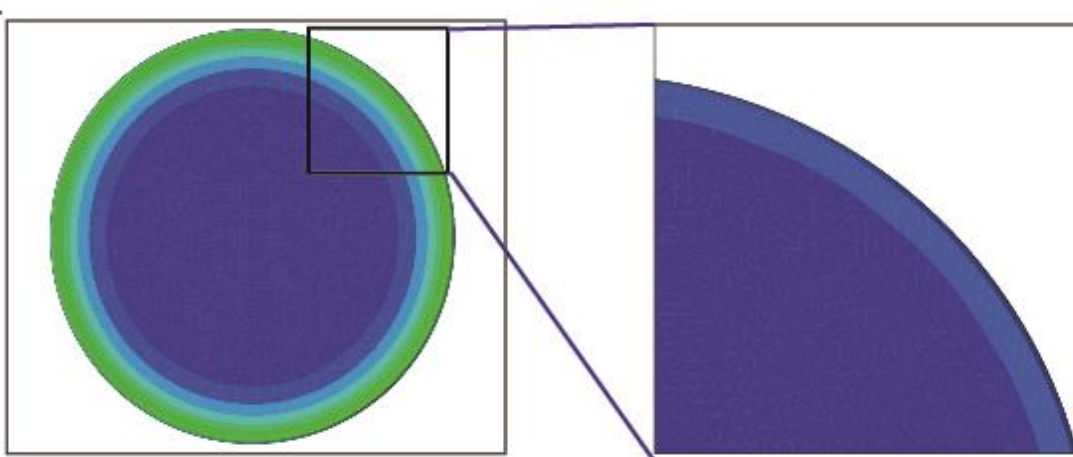

Strain Energy Density (fJ/um ${ }^{3}$ )

0.13
0.10
0.08
0.05
0.03
0.00 
(b) $t=960 \mathrm{~s}$

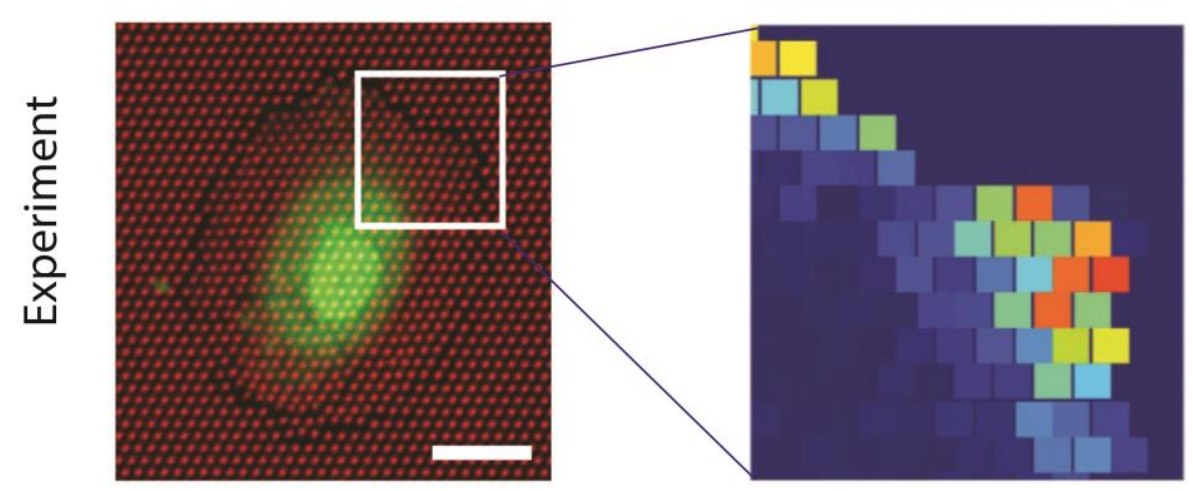

Strain Energy Density

(fJ/um ${ }^{3}$ )

0.13

0.10

0.08

0.05

0.03

0.00

Displacement

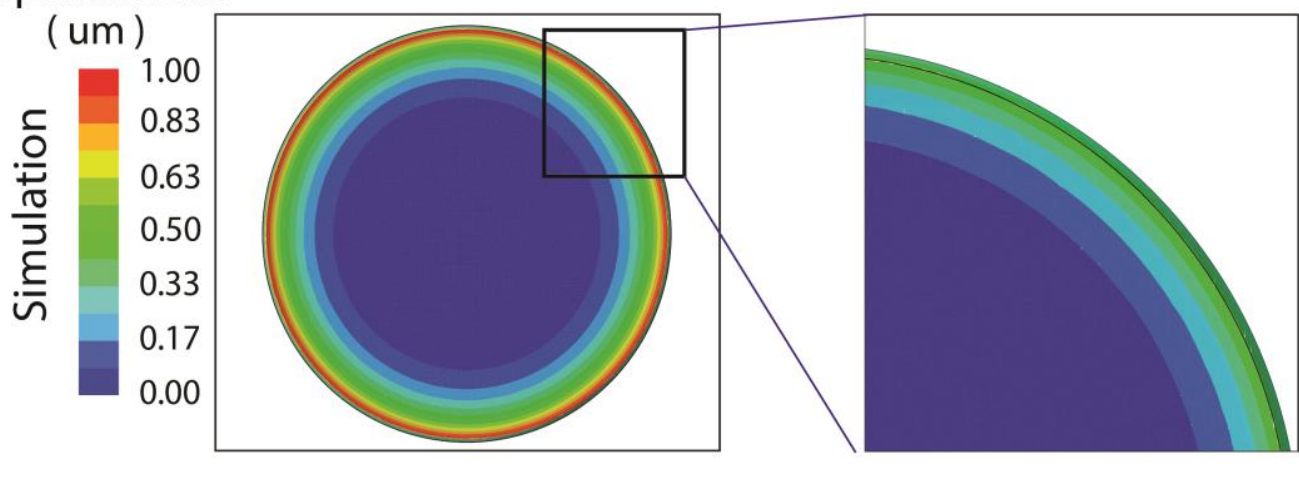

Strain Energy Density (fJ/um ${ }^{3}$ )

0.13
0.10
0.08
0.05
0.03
0.00

Figure 8 


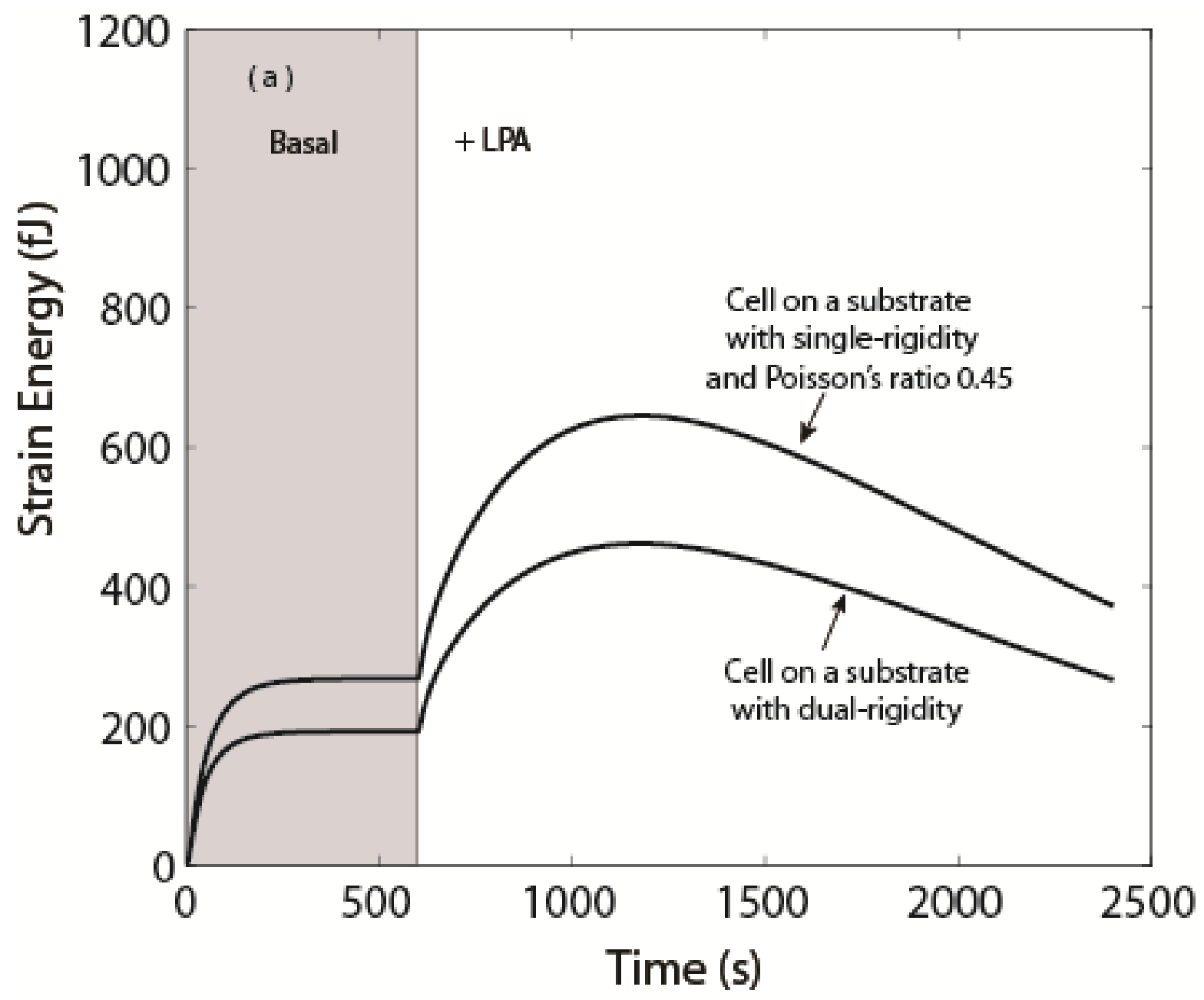

(b)
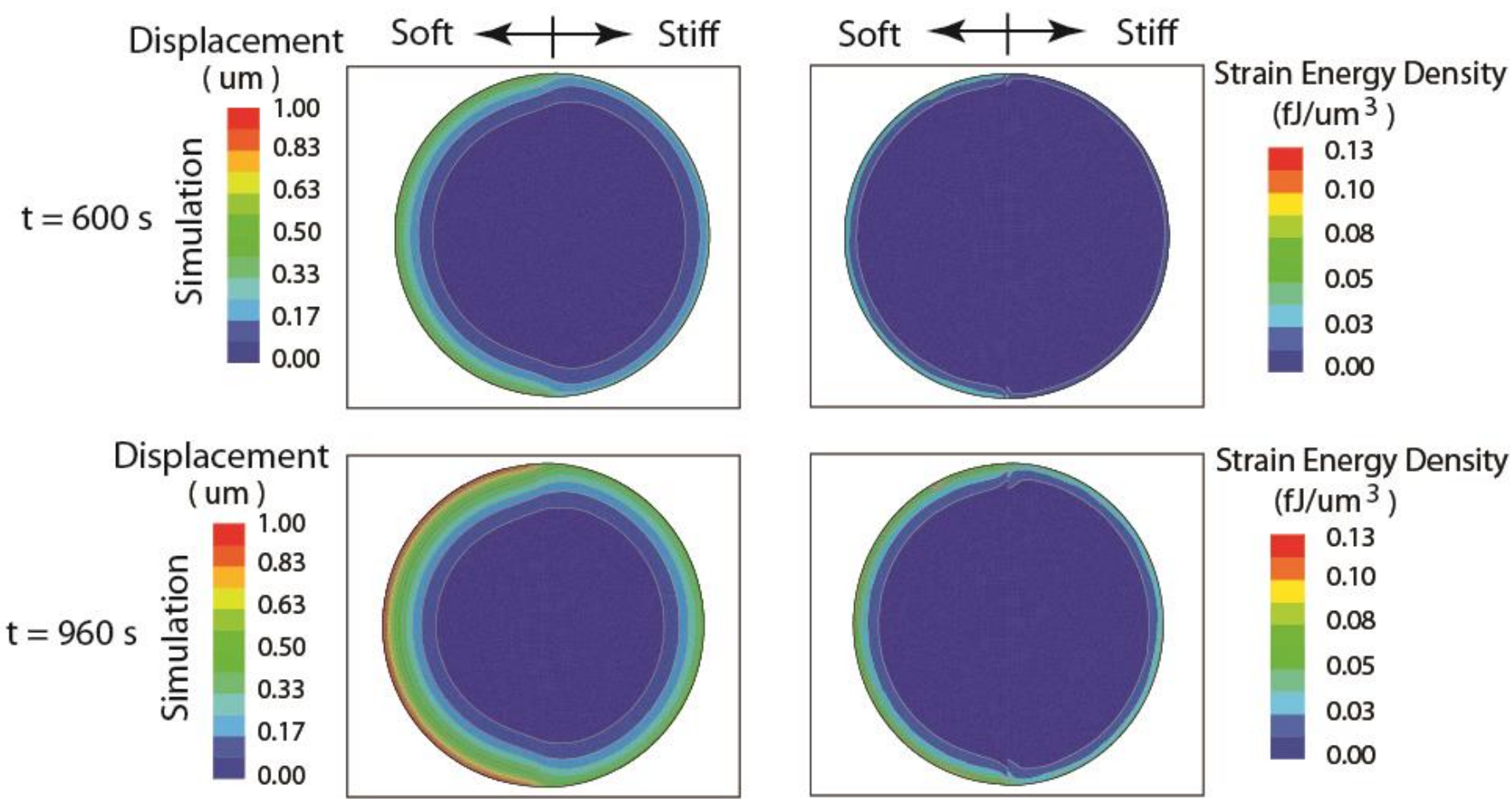

Strain Energy Density (fJ/um ${ }^{3}$ )

0.13
0.10
0.08
0.05
0.03
0.00


(c)
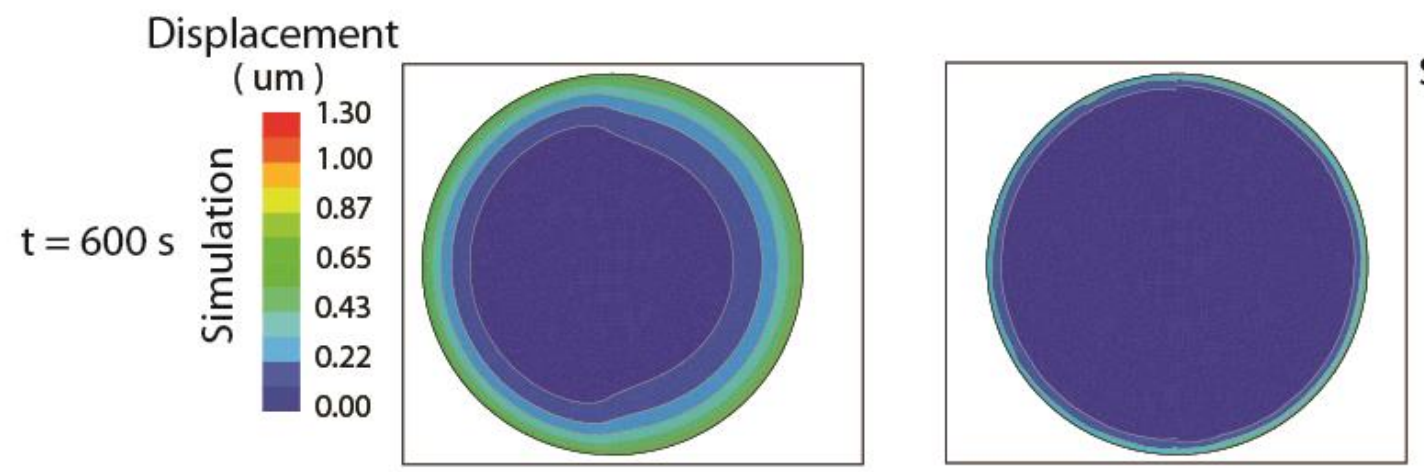

Strain Energy Density (fJ/um ${ }^{3}$ )

0.13

0.10

0.08

0.05

0.03
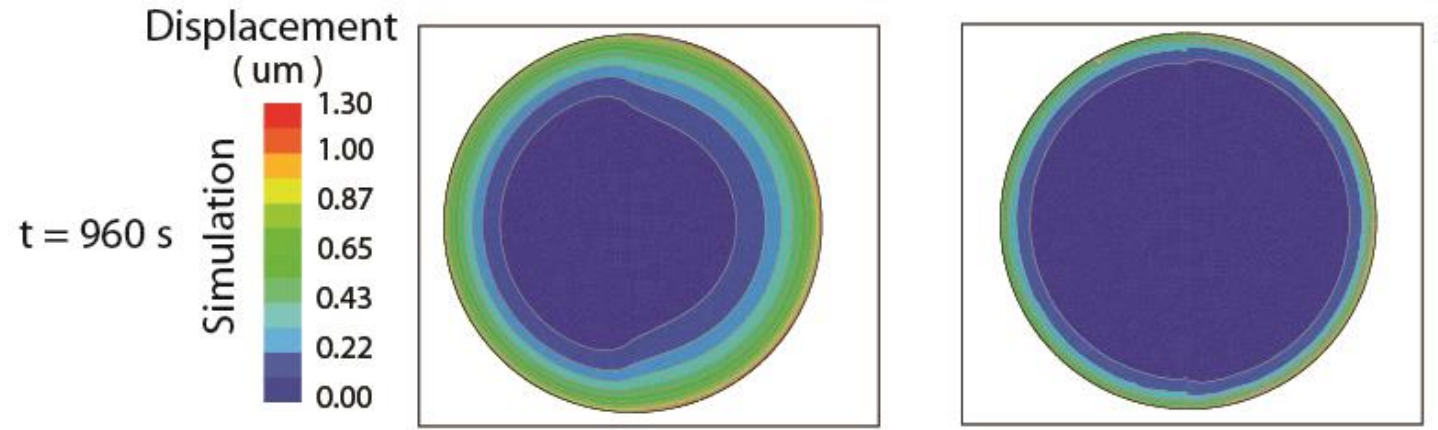

Strain Energy Density (fJ/ $\mathrm{um}^{3}$ )

0.13
0.10
0.08
0.05
0.03
0.00

Figure 9 